\title{
Tachyon Warm Intermediate and Logamediate Inflation in the Brane World Model in the Light of Planck Data
}

\author{
V. Kamali ${ }^{1}$ and M. R. Setare ${ }^{2}$ \\ ${ }^{1}$ Department of Physics, Faculty of Science, Bu-Ali Sina University, Hamedan 65178, Iran \\ ${ }^{2}$ Department of Science, University of Kurdistan, Campus of Bijar, Bijar 15175, Iran \\ Correspondence should be addressed to V. Kamali; vkamali1362@gmail.com
}

Received 4 April 2016; Revised 2 June 2016; Accepted 10 July 2016

Academic Editor: Vladimir Dzhunushaliev

Copyright (C) 2016 V. Kamali and M. R. Setare. This is an open access article distributed under the Creative Commons Attribution License, which permits unrestricted use, distribution, and reproduction in any medium, provided the original work is properly cited. The publication of this article was funded by SCOAP ${ }^{3}$.

Tachyon inflationary universe model on the brane in the context of warm inflation is studied. In slow-roll approximation and in longitudinal gauge, we find the primordial perturbation spectrums for this scenario. We also present the general expressions of the tensor-scalar ratio, scalar spectral index, and its running. We develop our model by using exponential potential; the characteristics of this model are calculated in great detail. We also study our model in the context of intermediate (where scale factor expands as $a=a_{0} \exp \left(A t^{f}\right)$ ) and logamediate (where the scale factor expands as $a=a_{0} \exp \left(A[\ln t]^{\nu}\right)$ ) models of inflation. In these two sectors, dissipative parameter is considered as a constant parameter and a function of tachyon field. Our model is compatible with observational data. The parameters of the model are restricted by Planck data.

\section{Introduction}

Inflation as a theoretical framework presents the better description of the early phase of our universe. Main problems of Big Bang model (horizon, flatness, etc.) could be solved in the context of inflation scenario [1,2]. Lagrangian formalism in terms of scalar fields can explain this scenario. Quantum fluctuations of the scalar field provide a description of anisotropy of cosmic microwave background (CMB) and origin of the distribution of large scale structure (LSS) [36]. Standard model of inflation, "cold inflation," has two regimes: slow-roll and (p)reheating. In the slow-roll limit kinematic energy is small compared to the potential energy term and the universe expands. Interaction between scalar field (inflation) and other fields (massive and radiation fields) is neglected. After this period, kinetic energy is comparable to the potential energy in (p)reheating epoch. In this era inflation oscillates around the minimum of the potential while losing their energy to other fields (radiation, massless fields) which are presented in the theory. After reheating, the universe is filled by radiation. In ( $p$ )reheating epoch, observed universe attaches to the end of inflationary period.
Another view of reheating is based on quantum mechanical production of massive particles in classical background inflation $[7,8]$. Preheating is probably the most efficient and plausible bridge that could connect inflation to a hot radiation dominated universe $[9,10]$.

In warm inflationary scenario radiation production occurs during the slow-roll inflation epoch and (p)reheating is avoided [11, 12]. In this scenario thermal fluctuations could play a dominant role to produce initial fluctuations which are necessary for LSS formation [13, 14]. Warm inflationary period ends when the universe stops inflating. After this period the universe enters in the radiation phase [11, 12]. Some extensions of this model are found in [15-19].

In warm inflation there has to be continuously particle production. For this to be possible, then the microscopic processes that produce these particles must occur at a timescale much faster than Hubble expansion. Thus the decay rates $\Gamma_{i}$ (not to be confused with the dissipative coefficient) must be bigger than $H$. Also these produced particles must thermalize. Thus the scattering processes amongst these produced particles must occur at a rate bigger than $H$. These adiabatic conditions were outlined since the early warm 
inflation papers, such as $[20,21]$. More recently there has been considerable explicit calculation from Quantum Field Theory (QFT) that explicitly computes all these relevant decay and scattering rates in warm inflation models $[22,23]$.

The inflation era in the early evolution of the universe could be described by tachyonic field, associated with unstable D-brane, because of the tachyon condensation near the maximum of the effective potential [24-26]. At the late times, tachyonic fields may add a nonrelativistic fluid or a new form of cosmological dark matter to the universe [27]. The tachyon inflation is a k-inflation model [28], for scalar field $\phi$ with a positive potential $V(\phi)$. Tachyon potentials have two special properties; firstly a maximum of this potential is obtained where $\phi \rightarrow 0$ and second property is the minimum of these potentials which is obtained where $\phi \rightarrow \infty$. If the tachyon field starts to roll down the potential, then universe dominated by a new form of matter will smoothly evolve from inflationary universe to an era which is dominated by a nonrelativistic fluid [27]. So, we can explain the phase of acceleration expansion (inflation) in terms of tachyon field. Tachyon fields in the ordinary (cold) tachyon inflation framework, after slow-roll epoch, evolve towards minimum of the potential without oscillating about it [26], so, the (p)reheating mechanism in cold tachyon inflation does not work. Warm tachyon inflation is a picture, where there are dissipative effects playing during inflation. As a result of this the inflation evolves in a thermal radiation bath; therefore the reheating problem of cold tachyon inflation [26] can be solved in the framework of warm tachyon inflation. We note that the cold tachyonic inflation era can naturally end with the collision of the two branes. In this situation we do not need warm inflation. If the collision of two branes does not arise naturally, warm inflation is perfectly good scenario that can solve the problem of end of thachyon inflation.

We may live on a brane which is embedded in a higher dimensional universe. This realization has significant implications to cosmology [29-34]. In this scenario, which is motivated by string theory, gravity (closed string modes) can propagate in the bulk, while the standard model of particles (matter fields which are related to open string modes) is confined to the lower-dimensional brane [35-37]. In terms of Randall-Sundrum suggestion, there are two similar but phenomenologically different brane world scenarios $[33,34]$. In this paper we will consider that the brane world model corresponds to the Randall-Sundrum II brane world [34].

The brane world picture is described by the following action [29-34]:

$$
\begin{aligned}
S= & \frac{1}{\kappa^{2}} \int d^{5} x \sqrt{-g}\left(R^{5}+\frac{12}{l^{2}}\right)-\lambda \int d^{4} x \sqrt{-g_{\text {brane }}} \\
& +\int d^{4} x \sqrt{-g_{\text {brane }}} L_{\text {matter }}
\end{aligned}
$$

In this scenario we have a 3-brane universe which is located in the 5D Anti-de Sitter (AdS) space-time, where this spacetime is effectively compactified with curvature scale $l$ of AdS space-time. $R^{5}$ is the Ricci scalar in five dimensions and $\kappa=8 \pi G_{5}=8 \pi / M_{5}^{3}$, where $G_{5}$ is the $5 \mathrm{D}$ Newton's constant and $M_{5}$ is Planck scale in five dimensions. $\lambda$ is the tension of the brane and if we have no matter on the brane $\kappa^{2} \lambda=6 / l$, where the brane becomes Minkowski spacetime. In the brane world model the gravity could propagate in the 5D space-time and the Newtonian gravity in four dimensions is reproduced at the scales larger than $l$ on the brane. 4D Einstein's equation projected onto the brane has been found in [38]. Friedmann equation and the equations of linear perturbation theory $[39,40]$ may be modified by these projections. Einstein's equations which are projected onto the brane with cosmological constant and matter fields which are confined to 3-brane tension have the following form [38]:

$$
G_{\mu \nu}=-\Lambda_{4} g_{\mu \nu}+\left(\frac{8 \pi}{M_{4}^{2}}\right) T_{\mu \nu}+\left(\frac{8 \pi}{M_{5}^{3}}\right)^{2} \pi_{\mu \nu}-E_{\mu \nu}
$$

where $E_{\mu \nu}$ is a projection of $5 \mathrm{D}$ weyl tensor, $T_{\mu \nu}$ is energy density tensor on the brane, $M_{4}=\sqrt{l M_{5}^{3}}$ is the Planck scales in $4 \mathrm{D}$, and $\pi_{\mu \nu}$ is a tensor quadratic in $T_{\mu \nu}$

$$
\begin{aligned}
\pi_{\mu \nu}= & -\frac{1}{4} T_{\mu \alpha} T_{\nu}^{\alpha}+\frac{1}{12} T_{\alpha}^{\alpha} T_{\mu \nu} \\
& +\frac{1}{24}\left(3 T_{\alpha \beta} T^{\alpha \beta}-\left(T_{\alpha}^{\alpha}\right)^{2}\right) g_{\mu \nu} .
\end{aligned}
$$

Cosmological constant $\Lambda_{4}$ on the brane in terms of 3brane tension $\lambda$ and $5 \mathrm{D}$ cosmological constant $\Lambda$ is given by

$$
\Lambda_{4}=\frac{4 \pi}{M_{5}^{3}}\left(\Lambda+\frac{4 \pi}{3 M_{5}^{3}} \lambda^{2}\right) .
$$

4D Planck scale is determined by 5D Planck scale as

$$
M_{4}=\sqrt{\frac{3}{4 \pi}}\left(\frac{M_{5}^{2}}{\sqrt{\lambda}}\right) M_{5}=\sqrt{\frac{4 \pi \lambda}{3}} l
$$

The natural boundary conditions to specify the perturbations of this model are imposed, where the perturbations do not diverge at the horizon of the AdS space-time and we assume that the Weyl curvature may be neglected. On the large scale, the behavior of cosmological perturbations on the brane world models is the same as a closed system on the brane without the effects of the perturbations along the extradimensions in the bulk [41-43]. On the large scale limit, the perturbation parameters of inflation models have a complete set of perturbed equations on the brane which may be solved in quasi-stable and slow-roll limit [41-44]. The study of the perturbation evolution of warm inflation in the brane world model on the large scale, by using equations solely on the brane and without solving the bulk perturbations, is found in [44]. This model has a complete set of perturbed equations on the brane. We would like to study the warm tachyon inflation model on the brane using this approach. Therefore we will consider the linear cosmological perturbations theory for warm tachyon inflation model on the brane. In spatially flat FRW model the Friedmann equation, by using Einstein's equation (3), has following form [38]:

$$
H^{2}=\frac{\Lambda_{4}}{3}+\left(\frac{8 \pi}{3 M_{4}^{2}}\right) \rho+\left(\frac{4 \pi}{3 M_{5}^{3}}\right) \rho^{2}+\frac{\varepsilon}{a^{4}},
$$


where $a$ is scale factor of the model and $H$ is Hubble parameter and $\rho$ is the total energy density on the brane. The last term in the above equation denotes the influence of the bulk gravitons on the brane, where $\varepsilon$ is an integration constant which arising from Weyl tensor $E_{\mu v}$. This term may be rapidly diluted once inflation begins and we will neglect it. Therefore the projected Weyl tensor term in the effective Einstein equation may be neglected and this term does not give the significant contributions to the observable perturbation parameters. We will also take $\Lambda_{4}$ to be vanished at least in the early universe. So, the Friedmann equation reduces to

$$
H^{2}=\frac{8 \pi}{3 M_{4}^{2}} \rho\left(1+\frac{\rho}{2 \lambda}\right) .
$$

The brane tension $\lambda$ has been constrained from nucleosynthesis [45] $\lambda>(1 \mathrm{MeV})^{4}$ and a stronger limit of it results from current tests for deviation from Newton's law, $\lambda \geq(10 \mathrm{TeV})^{4}$ $[46,47]$.

In the warm inflationary models the total energy density $\rho=\rho_{\phi}+\rho_{\gamma}$ is presented on the brane [48], where $\rho_{\gamma}$ is the energy density of the radiation. The Friedmann equation has this form

$$
H^{2}=\frac{8 \pi}{3 M_{4}^{2}}\left(\rho_{\phi}+\rho_{\gamma}\right)\left(1+\frac{\rho_{\phi}+\rho_{\gamma}}{2 \lambda}\right) .
$$

Cosmological perturbations of warm inflation model have been studied in [49]. Warm tachyon inflationary universe model has been studied in [50-53]; also warm inflation on the brane has been studied in [44]. Inflation era is located in a period of dynamical evolution of the universe that the effect of string/M-theory is relevant. On the other hand, string/M-theory is related to higher dimension theories such as space-like branes [24]. Therefore in the present work we will study warm tachyon inspired inflation in the context of a higher dimensional theory instead of General Relativity, that is, Randall-Sundrum brane world and cosmological perturbations of the model by using the above modified Einstein and Friedmann equations.

Recently there has been a new perspective of warm inflation [54] which is considered warm inflationary era as a quasi-de Sitter epoch of universe expansion; on the other hand as we mentioned it is believed that we may live on the brane; therefore we are interested to study warm tachyon inflation on the brane by using quasi-de Sitter solutions of scale factor.

In one sector of the present work, we would like to consider warm tachyon model on the brane in the context of "intermediate inflation." This scenario is one of the exact solutions of inflationary field equation in the Einstein theory with scale factor $a(t)=a_{0} \exp \left(A t^{f}\right)(A>0,0<f<1)$; this solution of the scale factor in the context of a modified tensor-scalar theory has been found in [55]. The study of this model is motivated by string/M-theory [56]. If we add the higher order curvature correction, which is proportional to Gauss-Bonnet (GB) term and to Einstein-Hilbert action then we obtain a free-ghost action [57, 58]. Gauss-Bonnet interaction is leading order of the " $\alpha$ " expansion to low energy string effective action $[57,58]$ ( $\alpha$ is inverse string tension). This theory may be applied for black hole solutions [59], acceleration of the late time universe $[60,61]$, and initial singularity problems [62]. The GB interaction in 4D with dynamical dilatonic scalar coupling leads to an intermediate form of scale factor [56]. Expansion of the universe in the intermediate inflation scenario is slower than standard de Sitter inflation with scale factor $a=a_{0} \exp \left(H_{0} t\right)\left(a_{0}, H_{0}>0\right)$ which arises as $f=1$, but faster than power-low inflation with scale factor $a=t^{p}(p>1)$. Harrison-Zeldovich [63-65] spectrum of density perturbation, that is, $n_{s}=1$ for intermediate inflation models driven by scalar field, is presented for exact values of parameter $f$ [66].

On the other hand we will also study our model in the context of "logamediate inflation" with scale factor $a(t)=$ $a_{0} \exp \left(a[\ln t]^{\nu}\right)(\nu>1, A>0)$ [67]. This model is converted to power-law inflation for $v=1$ cases. This scenario is applied in a number of scalar-tensor theories [68]. The study of logamediate scenario is motivated by imposing weak general conditions on the cosmological models which have indefinite expansion [67]. The effective potential of the logamediate model has been considered in dark energy models [69]. This form of potential is also used in supergravity, Kaluza-Klein theories, and superstring models $[68,70]$. For logamediate models the power spectrum could be either red or blue tilted [71, 72]. In [67], we can find eight possible asymptotic scale factor solutions for cosmological dynamics. Three of these solutions are noninflationary scale factor; another three solutions give power-low, de Sitter, and intermediate scale factors. Finally, two cases of these solutions have asymptotic expansion with logamediate scale factor. We will study our model using intermediate and logamediate scenarios.

Warm inflation models based on ordinary scalar fields have been studied in [15, 44, 73-77]. Particular model of warm inflation which is driven by tachyon field can be found in [50-53]. In [78], the consistency of warm tachyon inflation with viscous pressure has been studied and the stability analysis for that model has been done. In the present paper we will study warm tachyon inflation without viscosity effect on the brane. We also extended our model by using exact solutions of the scale factor by Barrow [67], that is, inter $(\log a)$ mediate solution.

The paper is organized as follows: in the next section we will describe warm tachyon inflationary universe model in the brane scenario in the background level. In Section 3 we present the perturbation parameters for our model. In Section 4 we study our model using the exponential potential in high dissipative regime and high energy limit. In Section 5 we study the model using intermediate scenario. In Section 6 we develop our model in the context of logamediate inflation. Finally in Section 7 we close by some concluding remarks.

\section{The Model}

Tachyon scalar field $\phi$ is described by relativistic Lagrangian [27] as

$$
L=\sqrt{-g}\left(\frac{R}{16 \pi G}-V(\phi) \sqrt{1-g^{\mu \nu} \partial_{\mu} \phi \partial_{\nu} \phi}\right) .
$$


The stress-energy tensor in a spatially flat Friedmann Robertson Walker (FRW) space-time is presented by

$$
T_{\nu}^{\mu}=\frac{\partial L}{\partial\left(\partial_{\mu} \phi\right)} \partial_{\nu} \phi-g_{\nu}^{\mu} L=\operatorname{diag}\left(-\rho_{\phi}, P_{\phi}, P_{\phi}, P_{\phi}\right) .
$$

From the above equation, energy density and pressure for a spatially homogeneous field have the following forms:

$$
\begin{aligned}
& \rho_{\phi}=\frac{V(\phi)}{\sqrt{1-\dot{\phi}^{2}}}, \\
& P_{\phi}=-V(\phi) \sqrt{1-\dot{\phi}^{2}},
\end{aligned}
$$

where $V(\phi)$ is a scalar potential associated with the tachyon field $\phi$. Important characteristics of this potential are $d V / d \phi<0$ and $V(\phi \rightarrow \infty) \rightarrow 0$ [79]. In this section, we will present the characteristics of warm tachyon inflation model on the brane in the background level. This model may be described by an effective fluid where the energy-momentum tensor of this fluid was recognized in the above equation.

The dynamic of the warm tachyon inflation in spatially flat FRW model on the brane is described by these equations:

$$
\begin{aligned}
& H^{2}=\frac{8 \pi}{3 M_{4}^{2}}\left[\frac{V(\phi)}{\sqrt{1-\dot{\phi}^{2}}}+\rho_{\gamma}\right] \\
& \cdot\left[1+\frac{1}{2 \lambda}\left(\frac{V(\phi)}{\sqrt{1-\dot{\phi}^{2}}}+\rho_{\gamma}\right)\right], \\
& \dot{\rho}_{\phi}+3 H\left(P_{\phi}+\rho_{\phi}\right)=-\Gamma \dot{\phi}^{2} \Longrightarrow \\
& \frac{\ddot{\phi}}{1-\dot{\phi}^{2}}+3 H \dot{\phi}+\frac{V^{\prime}}{V} \\
& =-\frac{\Gamma}{V} \sqrt{1-\dot{\phi}^{2}} \dot{\phi}^{\prime} \\
& \dot{\rho}_{\gamma}+4 H \rho_{\gamma}=\Gamma \dot{\phi}^{2},
\end{aligned}
$$

where $\Gamma$ is the dissipative coefficient. In the above equations dots "." mean derivative with respect to cosmic time and prime denotes derivative with respect to scalar field $\phi$. During slow-roll inflation era the energy density (11) is the order of potential $\rho_{\phi} \sim V$ and dominates over the radiation energy $\rho_{\phi}>\rho_{\gamma}$. Using the slow-roll limit when $\dot{\phi} \ll 1$ and $\ddot{\phi} \ll$ $(3 H+\Gamma / V) \dot{\phi}[11,12]$, and also when the inflation radiation production is quasi-stable $\left(\dot{\rho}_{\gamma} \ll 4 H \Gamma, \dot{\rho}_{\gamma} \ll \Gamma \dot{\phi}^{2}\right)$, the dynamic equations (12) and (13) are reduced to

$$
\begin{aligned}
H^{2} & =\frac{8 \pi}{3 M_{4}^{2}} V\left(1+\frac{V}{2 \lambda}\right), \\
3 H(1+r) \dot{\phi} & =-\frac{V^{\prime}}{V},
\end{aligned}
$$

where $r=\Gamma / 3 H V$. In canonical warm inflation scenario the relative strength of thermal damping $(\Gamma)$ should be compared to expansion damping $(H)$. We must analyse the warm inflation model in background and linear perturbation levels on our expanding over timescales which are shorter than the variation of expansion rate but large compared to the microphysical processes

$$
\begin{aligned}
& \frac{V}{\Gamma} \ll \tau \ll H^{-1} \Longrightarrow \\
& \Gamma \gg H V .
\end{aligned}
$$

For more discussion please see Appendix. Particle production in fact takes place at a constant rate during warm inflation for canonical scalar field where strength of thermal damping dominates over the effect of expansion damping $(\Gamma>H)$ but for tachyon scalar fields as presented in the above equation $\Gamma>H V$. We will study our model in high dissipative regime $(r \gg 1)$. Using these conditions we have $\Gamma \gg H V$ which agrees with particle production condition $(\Gamma>V H)$.

From (14), (15), and (16), $\rho_{\gamma}$ could be written as

$$
\rho_{\gamma}=\frac{\Gamma \dot{\phi}^{2}}{4 H}=\frac{M_{4}^{2} r}{32 \pi(1+r)^{2}(1+V / 2 \lambda)}\left(\frac{V^{\prime}}{V}\right)^{2}=\sigma T_{r}^{4},
$$

where $T_{r}$ is the temperature of thermal bath and $\sigma$ is StefanBoltzmann constant. We introduce the slow-roll parameters for our model as

$$
\begin{aligned}
\epsilon= & -\frac{\dot{H}}{H^{2}} \simeq \frac{M_{4}^{2}}{16 \pi} \frac{V^{\prime 2}}{(1+r) V^{3}} \frac{1+V / \lambda}{(1+V / 2 \lambda)^{2}}, \\
\eta= & -\frac{\ddot{H}}{H \dot{H}} \simeq \frac{M_{4}^{2}}{8 \pi} \frac{V^{\prime}}{V^{2}(1+r)[1+V / 2 \lambda]}\left[\frac{2 V^{\prime \prime}}{V^{\prime}}-\frac{V^{\prime}}{V}\right. \\
& \left.-\frac{r^{\prime}}{(1+r)}+\frac{V^{\prime}}{\lambda+V}\right]-2 \epsilon .
\end{aligned}
$$

A relation between two energy densities $\rho_{\phi}$ and $\rho_{\gamma}$ is obtained from (18) and (19)

$$
\begin{aligned}
\rho_{\gamma} & =\frac{r}{2(1+r)} \frac{\left[1+\rho_{\phi} / 2 \lambda\right]}{\left[1+\rho_{\phi} / \lambda\right]} \rho_{\phi} \epsilon \\
& \simeq \frac{r}{2(1+r)} \frac{[1+V / 2 \lambda]}{[1+V / \lambda]} V \epsilon .
\end{aligned}
$$

The condition of inflation epoch $\ddot{a}>0$ could be obtained by inequality $\epsilon<1$. Therefore from above equation, warm tachyon inflation on the brane could take place when

$$
\frac{2(1+r)}{r} \rho_{\gamma}<\frac{1+\rho_{\phi} / 2 \lambda}{1+\rho_{\phi} / \lambda} \rho_{\phi} .
$$

Inflation period ends when $\epsilon \simeq 1$ which implies

$$
\frac{M_{4}^{2}}{8 \pi}\left[\frac{V_{f}^{\prime}}{V_{f}}\right]^{2} \frac{1+V_{f} / \lambda}{\left(1+V_{f} / 2 \lambda\right)^{2}} \frac{1}{V_{f}} \simeq 2\left(1+r_{f}\right),
$$


where the subscript $f$ denotes the end of inflation. The number of e-folds is given by

$$
\begin{aligned}
N & =\int_{\phi_{*}}^{\phi_{f}} H d t=\int_{\phi_{*}}^{\phi_{f}} \frac{H}{\dot{\phi}} d \phi \\
& =-\frac{8 \pi}{M_{4}^{2}} \int_{\phi_{*}}^{\phi_{f}} \frac{V^{2}}{V^{\prime}}(1+r)\left[1+\frac{V}{2 \lambda}\right] d \phi,
\end{aligned}
$$

where the subscript $*$ denotes the epoch when the cosmological scale exits the horizon.

\section{Perturbation}

In this section we will study inhomogeneous perturbations of the FRW background. As we have mentioned in the introduction we ignore the influence of the bulk gravitons on the brane arising from Weyl tensor $E_{\mu \nu}$, so we neglect the back-reaction due to metric perturbations in the fifth dimension. These perturbations in the longitudinal gauge may be described by the perturbed FRW metric

$$
d s^{2}=(1+2 \Phi) d t^{2}-a^{2}(t)(1-2 \Psi) \delta_{i j} d x^{i} d x^{j}
$$

where $\Phi$ and $\Psi$ are gauge-invariant metric perturbation variables $[80,81]$. The equation of motion is given by

$$
\begin{aligned}
& \frac{\ddot{\delta} \phi}{1-\dot{\phi}^{2}}+\left[3 H+\frac{\Gamma}{V}\right] \dot{\delta} \phi \\
&+ {\left[-a^{-2} \nabla^{2}+\left(\frac{V^{\prime}}{V}\right)^{\prime}+\dot{\phi}\left(\frac{\Gamma}{V}\right)^{\prime}\right] \delta \phi } \\
&- {\left[\frac{1}{1-\dot{\phi}^{2}}+3\right] \dot{\phi} \dot{\Phi}-\left[\dot{\phi} \frac{\Gamma}{V}-2 \frac{V^{\prime}}{V}\right] \Phi=0 . }
\end{aligned}
$$

We expand the small change of field $\delta \phi$ into Fourier components as

$$
\begin{aligned}
\delta \phi & (x) \\
& =\int \frac{d^{3} k}{(2 \pi)^{3}}\left[e^{i k x} \delta \phi(k, t) a_{k}+e^{-i k x} \delta \phi(k, t) a_{k}^{*}\right] .
\end{aligned}
$$

In warm inflation thermal fluctuations of the inflation dominate over the quantum ones; therefore we have classical perturbation of scalar field $\delta \phi$. All perturbed quantities have a spatial sector of the form $e^{i k x}$, where $k$ is the wave number.
Perturbed Einstein field equations in momentum space have only the temporal parts

$$
\begin{aligned}
& \Phi=\Psi \\
& \dot{\Phi}+H \Phi=\frac{4 \pi}{M_{4}^{2}}\left[-\frac{4 \rho_{\gamma} a v}{3 k}+\frac{V \dot{\phi}}{\sqrt{1-\dot{\phi}^{2}}} \delta \phi\right] \\
& .\left[1+\frac{1}{\lambda}\left[\rho_{\gamma}+\frac{V}{\left.\left.\sqrt{1-\dot{\phi}^{2}}\right]\right]}\right.\right. \\
& \frac{\dot{\delta \phi}}{1-\dot{\phi}^{2}}+\left[3 H+\frac{\Gamma}{V}\right] \dot{\delta \phi}+\left[\frac{k^{2}}{a^{2}}+\left(\frac{V^{\prime}}{V}\right)^{\prime}+\dot{\phi}\left(\frac{\Gamma}{V}\right)^{\prime}\right] \\
& \cdot \delta \phi-\left[\frac{1}{1-\dot{\phi}^{2}}+3\right] \dot{\phi} \dot{\Phi}-\left[\dot{\phi} \frac{\Gamma}{V}-2 \frac{V^{\prime}}{V}\right] \Phi=0, \\
& \left(\dot{\delta \rho_{\gamma}}\right)+4 H \delta \rho_{\gamma}+\frac{4}{3} k a \rho_{\gamma} v-4 \rho_{\gamma} \dot{\Phi}-\dot{\phi}^{2} \Gamma^{\prime} \delta \phi \\
& -\Gamma \dot{\phi}^{2}[2(\dot{\delta \phi})-3 \dot{\phi} \Phi]=0, \\
& \dot{v}+4 H v+\frac{k}{a}\left[\Phi+\frac{\delta \rho_{\gamma}}{4 \rho_{\gamma}}+\frac{3 \Gamma \dot{\phi}}{4 \rho_{\gamma}} \delta \phi\right]=0 .
\end{aligned}
$$

The above equations are obtained for Fourier components $e^{i k x}$, where the subscript $k$ is omitted. $v$ in the above set of equations is presented by the decomposition of the velocity field $\left(\delta u_{j}=-\left(i a k_{J} / k\right) v e^{i k x}, j=1,2,3\right)[80,81]$.

Note that the effect of the bulk (extradimension) to perturbed projected Einstein field equations on the brane may be found in (29). We will describe the nondecreasing adiabatic and isocurvature modes of our model on large scale limit. In this limit we have obtained a complete set of perturbation equations on the brane. Therefore the perturbation variables along the extradimensions in the bulk could not have any contribution to the perturbation equations on super-horizon scales (see, e.g., [41-44]). The same approach, for nontachyon warm inflation model on the brane, in [44] is presented. Warm inflation model may be considered as a hybrid-like inflationary model where the inflation field interacts with radiation field $[49,82,83]$. Entropy perturbation may be related to dissipation term [84]. Perturbation of entropy in warm inflation model is given by [85]

$$
\delta S=e=-V_{, \phi T} \delta \phi-V_{, T T} \delta T .
$$

In this paper we will study potential of the model as a function of scalar field $(V(\phi))$; therefore the entropy perturbation will be neglected. We will study this important issue (potential as function of temperature, $V(\phi, T))$ in future works.

During inflationary phase with slow-roll approximation, for nondecreasing adiabatic modes on large scale limit $k \ll$ $a H$, we assume that the perturbed quantities could not vary strongly. So we have $H \Phi \gg \dot{\Phi},(\ddot{\delta} \phi) \ll(\Gamma+3 H)(\dot{\delta} \phi),\left(\dot{\delta} \dot{\rho}_{\gamma}\right) \ll$ $\delta \rho_{\gamma}$, and $\dot{v} \ll 4 H v$. In the slow-roll limit and by using the 
above limitations, the set of perturbed equations are reduced to

$$
\begin{aligned}
& \Phi \simeq \frac{4 \pi}{H M_{4}^{2}}\left[-\frac{4 \rho_{\gamma} a v}{3 k}+V \dot{\phi} \delta \phi\right]\left[1+\frac{V}{\lambda}\right], \\
& {\left[3 H+\frac{\Gamma}{V}\right] \dot{\delta} \phi+\left[\left(\frac{V^{\prime}}{V}\right)^{\prime}+\dot{\phi}\left(\frac{\Gamma}{V}\right)^{\prime}\right] \delta \phi } \\
& \simeq\left[\dot{\phi} \frac{\Gamma}{V}-2\left(\frac{V^{\prime}}{V}\right)\right] \Phi, \\
& \frac{\delta \rho_{\gamma}}{\rho_{\gamma}} \simeq \frac{\Gamma^{\prime}}{\Gamma} \delta \phi-3 \Phi, \\
& v \simeq-\frac{k}{4 a H}\left(\Phi+\frac{\delta \rho_{\gamma}}{4 \rho_{\gamma}}+\frac{3 \Gamma \dot{\phi}}{4 \rho_{\gamma}} \delta \phi\right) .
\end{aligned}
$$

Using (34), (36), and (37), perturbation variable $\Phi$ is determined

$\Phi$

$$
=\frac{4 \pi}{M_{4}^{2}}\left(\frac{V \dot{\phi}}{H}\right)\left[1+\frac{\Gamma}{4 H V}+\frac{\Gamma^{\prime} \dot{\phi}}{48 H^{2} V}\right]\left(1+\frac{V}{\lambda}\right) \delta \phi .
$$

We can solve the above equations by taking tachyon field $\phi$ as the independent variable in place of cosmic time $t$. Using (16) we find

$$
\left(3 H+\frac{\Gamma}{V}\right) \frac{d}{d t}=\left(3 H+\frac{\Gamma}{V}\right) \dot{\phi} \frac{d}{d \phi}=-\frac{V^{\prime}}{V} \frac{d}{d \phi} .
$$

From above equation, (35) and (38), the expression $(\delta \phi)^{\prime} / \delta \phi$ is obtained

$$
\begin{aligned}
& \frac{(\delta \phi)^{\prime}}{\delta \phi}=\frac{V}{V^{\prime}}\left[\left(\frac{V^{\prime}}{V}\right)^{\prime}+\dot{\phi}\left(\frac{\Gamma}{V}\right)^{\prime}\right. \\
& +\frac{4 \pi}{M_{4}^{2}}\left(-\dot{\phi} \frac{\Gamma}{V}+2\left(\frac{V^{\prime}}{V}\right)^{\prime}\right)\left(\frac{V \dot{\phi}}{H}\right) \\
& \left..\left[1+\frac{\Gamma}{4 H V}+\frac{\Gamma^{\prime} \dot{\phi}}{48 H^{2} V}\right]\left(1+\frac{V}{\lambda}\right)\right] .
\end{aligned}
$$

We will return to the above relation. Following [44, 50$53,84]$, we introduce auxiliary function $\chi$ as

$$
\chi=\frac{V \delta \phi}{V^{\prime}} \exp \left[\int \frac{1}{3 H+\Gamma / V}\left(\frac{\Gamma}{V}\right)^{\prime} d \phi\right] .
$$

From above definition we have

$$
\frac{\chi^{\prime}}{\chi}=\frac{(\delta \phi)^{\prime}}{\delta \phi}-\frac{V}{V^{\prime}}\left(\frac{V^{\prime}}{V}\right)^{\prime}+\frac{(\Gamma / V)^{\prime}}{3 H+\Gamma / V} .
$$

Using above equation and (40), we find

$$
\begin{aligned}
\frac{\chi^{\prime}}{\chi}= & \frac{4 \pi}{M_{4}^{2}}\left(-\frac{V \dot{\phi}}{V^{\prime}} \frac{\Gamma}{V}+2\right)\left(\frac{V \dot{\phi}}{H}\right) \\
& \cdot\left[1+\frac{\Gamma}{4 H V}+\frac{\Gamma^{\prime} \dot{\phi}}{48 H^{2} V}\right]\left(1+\frac{V}{\lambda}\right) .
\end{aligned}
$$

We could rewrite this equation, using (15) and (16)

$$
\begin{aligned}
\frac{\chi^{\prime}}{\chi} & =-\frac{9}{8} \\
& \cdot \frac{2 H+\Gamma / V}{(3 H+\Gamma / V)^{2}}\left(\Gamma+4 H V-\frac{\Gamma^{\prime} V^{\prime} / V}{12 H(3 H+\Gamma / V)}\right) \\
& \cdot \frac{V^{\prime}}{V^{2}} \frac{[1+V / \lambda]}{1+V / 2 \lambda} .
\end{aligned}
$$

A solution for the above equation is

$$
\chi(\phi)=C \exp \left(-\int\left\{-\frac{9}{8} \frac{2 H+\Gamma / V}{(3 H+\Gamma / V)^{2}}\left(\Gamma+4 H V-\frac{\Gamma^{\prime} V^{\prime} / V}{12 H(3 H+\Gamma / V)}\right) \frac{V^{\prime}}{V^{2}} \frac{[1+V / \lambda]}{1+V / 2 \lambda}\right\} d \phi\right),
$$

where $C$ is integration constant. From above equation and (42) we find small change of variable $\delta \phi$ as

$$
\delta \phi=C \frac{V^{\prime}}{V} \exp (\Im(\phi)),
$$

where

$$
\mathfrak{I}(\phi)=-\int\left[\frac{(\Gamma / V)^{\prime}}{3 H+\Gamma / V}+\left(\frac{9}{8}\right.\right.
$$

$$
\begin{aligned}
& \cdot \frac{2 H+\Gamma / V}{(3 H+\Gamma / V)^{2}}\left(\Gamma+4 H V-\frac{\Gamma^{\prime} V^{\prime} / V}{12 H(3 H+\Gamma / V)}\right) \\
& \left.\left.\cdot \frac{V^{\prime}}{V^{2}} \frac{[1+V / \lambda]}{1+V / 2 \lambda}\right)\right] d \phi .
\end{aligned}
$$

In the above calculations we have used the perturbation methods in warm inflation models [44, 50-53, 84], where 
the small change of variable $\delta \phi$ may be generated by thermal fluctuations instead of quantum fluctuations [86], and the integration constant $C$ may be driven by boundary conditions for field perturbation. Perturbed matter fields of our model are inflation $\delta \phi$, radiation $\delta \rho_{r}$, and velocity $k^{-1}(P+\rho) v_{, i}$. We can explain the cosmological perturbations in terms of gauge-invariant variables. These variables are important for development of perturbation after the end of inflation period. The curvature perturbation $\mathfrak{R}$ and entropy perturbation $e$ are defied by $[87,88]$

$$
\begin{gathered}
\Re=\Phi-k^{-1} a H v \\
e=\delta P-c_{s}^{2} \delta \rho,
\end{gathered}
$$

where $c_{s}^{2}=\dot{P} / \dot{\rho}$. The boundary condition of warm inflation models is found in very large scale limits; that is, $k \ll a H$ where the curvature perturbation $\mathfrak{R} \sim$ const and the entropy perturbation vanishes [85].

Finally the density perturbation is given by $[89,90]$

$$
\begin{aligned}
\delta_{H} & =\frac{2}{5} M_{4}^{2} \frac{V \exp (-\mathfrak{I}(\phi))}{V^{\prime}} \delta \phi \\
& =\frac{2}{15} M_{4}^{2} \frac{\exp (-\mathfrak{J}(\phi))}{H r \dot{\phi}} \delta \phi .
\end{aligned}
$$

For high or low energy limit $(V \gg \lambda$ or $V \ll \lambda)$ and by inserting $\Gamma=0$, the above equation reduces to $\delta_{H} \simeq(H / \dot{\phi}) \delta \phi$ which agrees with the density perturbation in cold inflation model $[1,2]$. In the warm inflation model the fluctuations of the scalar field in high dissipative regime $(r \gg 1)$ may be generated by thermal fluctuation instead of quantum fluctuations [86] as

$$
(\delta \phi)^{2} \simeq \frac{k_{F} T_{r}}{2 \pi^{2}}
$$

where in this limit freeze-out wave number $k_{F}=\sqrt{\Gamma H / V}=$ $H \sqrt{3 r} \geq H$ corresponds to the freeze-out scale at the point when dissipation damps out to thermally excited fluctuations $\left(V^{\prime \prime} / V^{\prime}<\Gamma H / V\right)[86] . \delta \phi$ in (50) can be found in [86], where Fourier transformed to momentum space is used (see, e.g., Appendix of [86] and Section 4 of [66]); therefore $\delta \phi$ is introduced in Fourier space and we can present spectral index and running in Fourier space. With the help of (49) and (50) in high energy $(V \gg \lambda)$ and high dissipative regime $(r \gg 1)$ we find

$$
\delta_{H}^{2}=\frac{2 \sqrt{3}}{75 \pi^{2}} M_{4}^{4} \frac{\exp (-2 \widetilde{\Im}(\phi))}{\sqrt{r} \widetilde{\epsilon}} \frac{T_{r}}{H}
$$

or equivalently

$$
\delta_{H}^{2}=\frac{4 M_{4}^{5} \lambda^{1 / 2}}{25(2 \pi)^{5 / 2} \sigma^{1 / 4}} V^{-3 / 4} r^{-1 / 2} \epsilon^{-3 / 4} \exp (-2 \widetilde{\Im}(\phi))
$$

where

$$
\begin{aligned}
\widetilde{\Im}(\phi)=-\int\left[\frac{1}{3 H r}\left(\frac{\Gamma}{V}\right)^{\prime}\right. \\
\left.+\frac{9}{4}\left(1-\frac{(\ln \Gamma)^{\prime} V^{\prime} / V}{36 r H^{2}}\right) \frac{V^{\prime}}{V}\right] d \phi, \\
\widetilde{\epsilon}=\frac{M_{4}^{2} \lambda}{4 \pi r} \frac{V^{\prime 2}}{V^{4}} .
\end{aligned}
$$

An important perturbation parameter of inflation models is scalar index $n_{s}$ which in high dissipative regime is presented by

$$
\begin{aligned}
n_{s} & =1+\frac{d \ln \delta_{H}^{2}}{d \ln k} \\
& \approx 1-\frac{3}{4} \widetilde{\epsilon}+\frac{3}{4} \widetilde{\eta}+\widetilde{\epsilon}\left(\frac{V}{V^{\prime}}\right)\left(2 \widetilde{\Im}^{\prime}(\phi)+\frac{r^{\prime}}{2 r}\right),
\end{aligned}
$$

where

$$
\widetilde{\eta}=\frac{M_{4}^{2} \lambda}{4 \pi r} \frac{V^{\prime}}{V^{3}}\left[\frac{2 V^{\prime \prime}}{V^{\prime}}-\frac{r^{\prime}}{r}\right]-2 \widetilde{\epsilon} .
$$

In (55) we have used a relation between small change of the number of e-folds and interval in wave number $(d N=$ $-d \ln k$ ). Running of the scalar spectral index may be found as

$$
\alpha_{s}=\frac{d n_{s}}{d \ln k}=-\frac{d n_{s}}{d N}=-\frac{d \phi}{d N} \frac{d n_{s}}{d \phi}=\frac{M_{4}^{2} \lambda}{4 \pi r} \frac{V^{\prime} n_{s}^{\prime}}{V^{3}} .
$$

This parameter is one of the interesting cosmological perturbation parameters which is approximately -0.038 , by using observational results $[3,4]$. During inflation epoch, there are two independent components of gravitational waves $\left(h_{x+}\right)$ with action of massless scalar field which are produced by the generation of tensor perturbations. Tensor perturbations do not couple to the thermal background; therefore gravitational waves are only generated by quantum fluctuations, the same as in standard fluctuations [86]. However, if the gravitational sector is modified then the expression for tensor power spectrum changes with respect to General Relativity. In particular, the amplitude of the tensor perturbation on the brane is presented as $[91,92]$

$$
A_{g}^{2}=\frac{16 \pi}{M_{4}^{4}}\left(\frac{H}{2 \pi}\right)^{2} F^{2}(x)=\frac{16}{3 M_{4}^{2} \lambda} V^{2} F^{2}(x),
$$

where the temperature $T$ in extra factor $\operatorname{coth}[k / 2 T]$ denotes the temperature of the thermal background of gravitational wave [93], $x=\left[3 H^{2} M_{4}^{2} / 4 \pi \lambda\right]^{1 / 2}$, and $F(x)=\left\{\sqrt{1+x^{2}}-\right.$ $\left.x^{2} \sinh ^{-1}(1 / x)\right\}^{-1 / 2}$ (in high energy limit, $V \gg \lambda$, we have $\left.F(x)=\left[27 M_{4}^{2} / 16 \pi \lambda\right]^{1 / 4} H^{1 / 2}=\left[3 \pi / \lambda^{3} M_{4}^{2}\right]^{1 / 4} V^{1 / 2}\right)$. Spectral index $n_{g}$ is presented as

$$
n_{g}=\frac{d}{d \ln k}\left(\ln \left[\frac{A_{g}^{2}}{\operatorname{coth}(k / 2 T)}\right]\right) \simeq-2 \widetilde{\epsilon}
$$


where $A_{g} \propto k^{n_{g}} \operatorname{coth}[k / 2 T]$ [93]. Using (51) and (58) we write the tensor-scalar ratio in high dissipative regime

$$
\begin{aligned}
& R(k)=\left.\frac{A_{g}^{2}}{P_{R}}\right|_{k=k_{0}}=\frac{16.2^{5 / 2} \pi^{11 / 4} \sigma^{1 / 4} V^{13 / 4} r^{1 / 2} \epsilon^{3 / 4}}{3^{3 / 4} \cdot M_{4}^{15 / 2} \lambda^{9 / 4}} \\
& \cdot \exp (2 \widetilde{\Im}(\phi)) \operatorname{coth}\left(\frac{k}{2 T}\right),
\end{aligned}
$$

where $k_{0}$ is referred to pivot point [93] and $P_{R}=(25 / 4) \delta_{H}^{2}$. An upper bound for this parameter is given by using Planck data, $R<0.11[3,4]$.

\section{Exponential Potential}

In this section we consider our model with the tachyonic effective potential

$$
V(\phi)=V_{0} \exp (-\alpha \phi)
$$

where parameter $\alpha>0$ is related to mass of tachyon field [94]. The exponential form of the potential has characteristics of tachyon field $\left(d V / d \phi<0\right.$ and $\left.V(\phi \rightarrow 0) \rightarrow V_{\max }\right)$. We develop our model in high dissipative regime; that is, $r \gg 1$ and high energy limit; that is, $V \gg \lambda$ for a constant dissipation coefficient $\Gamma$. From (54) slow-roll parameter $\widetilde{\epsilon}$ in the present case has the form

$$
\widetilde{\epsilon}=\frac{M_{4}^{2} \lambda}{8 \pi} \frac{\alpha^{2}}{r V_{0}^{2} e^{-2 \alpha \phi}} .
$$

Also the other slow-roll parameter $\widetilde{\eta}$ is obtained from (56)

$$
\widetilde{\eta}=-\frac{M_{4}^{2}}{4 \pi} \frac{\alpha^{2}}{r V_{0}^{2} e^{-2 \alpha \phi}} .
$$

Dissipation parameter $r=\Gamma / 3 H V$ in this case is given by

$$
r=\sqrt{\frac{\Gamma_{0}^{2} M_{4}^{2} \lambda}{12 \pi}} \frac{e^{2 \alpha \phi}}{V_{0}^{2}} .
$$

We find the evolution of tachyon field with the help of (16)

$$
\phi(t)=\frac{1}{\alpha} \ln \left[\frac{\alpha^{2} V_{0}}{\Gamma_{0}} t+e^{\alpha \phi_{i}}\right],
$$

where $\phi_{i}=\phi(t=0)$. Hubble parameter for our model has this form

$$
H=\sqrt{\frac{4 \pi}{3 M_{4}^{2} \lambda}} V_{0} e^{-\alpha \phi} .
$$

Using (21) and (62), the energy density of the radiation field in high dissipative limit becomes

$$
\rho_{\gamma}=\frac{3 M_{4} \alpha^{2}}{16 \Gamma_{0}} \frac{V_{0}^{2}}{\sqrt{3 \pi \lambda}} e^{-2 \alpha \phi}
$$

and in terms of tachyon field energy density $\rho_{\phi}$ becomes

$$
\rho_{\gamma}=\frac{3 M_{4}^{2}}{16 \sqrt{3 \pi \lambda}}\left(\frac{\alpha^{2}}{\Gamma_{0}}\right) \rho_{\phi}^{2} .
$$

From (24) the number of e-folds, at the end of inflation, by using the potential (61) for our inflation model is presented by

$$
N_{\text {total }}=\sqrt{\frac{4 \pi \lambda}{3 M_{4}^{2}}} \frac{\Gamma_{0}}{\alpha}\left(\phi_{f}-\phi_{i}\right)
$$

or equivalently

$$
N_{\text {total }}=\sqrt{\frac{4 \pi \lambda}{3 M_{4}^{2}}} \frac{\Gamma_{0}}{\alpha^{2}} \ln \left(\frac{V_{i}}{V_{f}}\right),
$$

where $V_{i}>V_{f}$. Using (51) and (60), we could find the scalar spectrum and scalar-tensor ratio

$$
\delta_{H}^{2}=A \exp \left(-\frac{7}{2} \alpha \phi\right)
$$

where $A=(16 \sqrt{3} / 75 \pi)\left(V_{0}^{3 / 2} M_{4}^{2} / \alpha^{2}\right)\left(\Gamma_{0}^{2} M_{4}^{2} \lambda / 12 \pi\right)^{1 / 2}\left(3 M_{4}^{2} \lambda /\right.$ $4 \pi)^{1 / 4}$ and

$$
R=B \exp (-\alpha \phi)
$$

where $B=\left(50 \pi^{3 / 2} \alpha^{2} V_{0} / 3 M_{4}^{7} \lambda^{3 / 2} T_{r}\right)\left(36 \pi^{2} / \Gamma_{0}^{2} M_{4}^{4} \lambda^{4}\right)^{1 / 4}$. In the above equation we have used (53), where

$$
\widetilde{\mathfrak{\Im}}(\phi)=-\frac{5}{4} \ln V
$$

These parameters may by restricted by Planck observational data [3-6].

\section{Intermediate Inflation}

Intermediate inflation is denoted by the scale factor

$$
a(t)=a_{0} \exp \left(A t^{f}\right), \quad 0<f<1 .
$$

This model of inflation is faster than power-low inflation and slower than de Sitter inflation. In this section we will study our model in the context of intermediate inflation in two cases: (1) $\Gamma=\Gamma_{0}$ and (2) $\Gamma=\Gamma_{1} V(\phi)$ which have been considered in the literature [50-53].

5.1. $\Gamma=\Gamma_{0}$ Case. In high dissipative $(r \gg 1)$ and high energy $(V \gg \lambda)$ limits the equations of the slow-roll inflation, that is, (12) and (13), are simplified as

$$
\begin{aligned}
V & =\left(\frac{3 \lambda M_{4}^{2}}{4 \pi}\right)^{1 / 2} H, \\
\dot{\phi}^{2} & =-\frac{\dot{V}}{\Gamma} .
\end{aligned}
$$


Inflation field may be derived from above equations in this case $\left(\Gamma=\Gamma_{0}\right)$

$$
\phi-\phi_{0}=\beta t^{f / 2},
$$

where $\beta=\left(12 \lambda M_{4}^{2} A^{2}(1-f)^{2} / \pi f^{2} \Gamma_{0}^{2}\right)$. Using above equation and the scale factor of intermediate inflation, tachyonic potential and Hubble parameter are presented as

$$
\begin{aligned}
& H(\phi)=f A\left(\frac{\phi-\phi_{0}}{\beta}\right)^{(2 f-2) / f}, \\
& V(\phi)=\left(\frac{3 \lambda M_{4}^{2} f^{2} A^{2}}{4 \pi}\right)^{1 / 2}\left(\frac{\phi-\phi_{0}}{\beta}\right)^{(2 f-2) / f} .
\end{aligned}
$$

Dissipative parameter $r$ is given by using above equation

$$
r=\frac{\Gamma_{0}}{3 H V}=\frac{4 \pi \Gamma_{0}}{9(f A)^{2} M_{4}^{2} \lambda}\left(\frac{\phi-\phi_{0}}{\beta}\right)^{(4-4 f) / f} .
$$

The slow-roll parameters of the model in the present case may be obtained as

$$
\begin{aligned}
& \epsilon=-\frac{\dot{H}}{H^{2}}=\frac{1-f}{f A}\left(\frac{\phi-\phi_{0}}{\beta}\right)^{-2}, \\
& \eta=-\frac{\ddot{H}}{\dot{H} H}=\frac{2-f}{f A}\left(\frac{\phi-\phi_{0}}{\beta}\right)^{-2} .
\end{aligned}
$$

We present the number of e-folds as

$$
N=\int_{t_{1}}^{t} H d t=A\left(\left[\frac{\phi-\phi_{0}}{\beta}\right]^{2}-\left[\frac{\phi_{1}-\phi_{0}}{\beta}\right]^{2}\right),
$$

where $\phi_{1}=\phi_{0}+\beta((1-f) / f A)^{1 / 2}$ is the scalar field at the beginning of the inflation. From the above equation we can present the scalar field in terms of number of e-folds and intermediate parameters

$$
\phi=\beta\left(\frac{N}{A}+\frac{1-f}{f A}\right)^{1 / 2}+\phi_{0} .
$$

Now we could find the perturbation parameters of the model. The power spectrum is obtained from (51), (53), and (73)

$$
\begin{aligned}
P_{R} & =\frac{25}{4} \delta_{H}^{2}=\frac{M_{4}^{5} \lambda^{1 / 2}}{(2 \pi)^{5 / 2} \sigma^{1 / 4}} \frac{V^{7 / 4}}{r^{1 / 2} \epsilon^{3 / 4}} \\
& =A_{1}\left(\frac{\phi-\phi_{0}}{\beta}\right)^{(14 f-11) / 2 f} \\
& =A_{1}\left(\frac{N}{A}+\frac{1-f}{f A}\right)^{(14 f-11) / 4 f},
\end{aligned}
$$

where $A_{1}=2^{5 / 2} M_{4}^{31 / 4}(3 \lambda)^{15 / 8}(f A)^{7 / 2} /(4 \pi)^{31 / 8} \sigma^{1 / 4} \Gamma_{0}^{1 / 2}(1-$ $f)^{3 / 4}$. We present the spectral index $n_{s}$ which is one of the important perturbation parameters from (55) and (73)

$$
\begin{aligned}
n_{s} & =1+\frac{3}{4} \eta-\frac{17}{4} \epsilon=1-\frac{11-14 f}{4 f A}\left(\frac{\phi-\phi_{0}}{\beta}\right)^{-2} \\
& =1-\frac{11-14 f}{4 f A}\left(\frac{N}{A}+\frac{1-f}{f A}\right)^{-1} .
\end{aligned}
$$

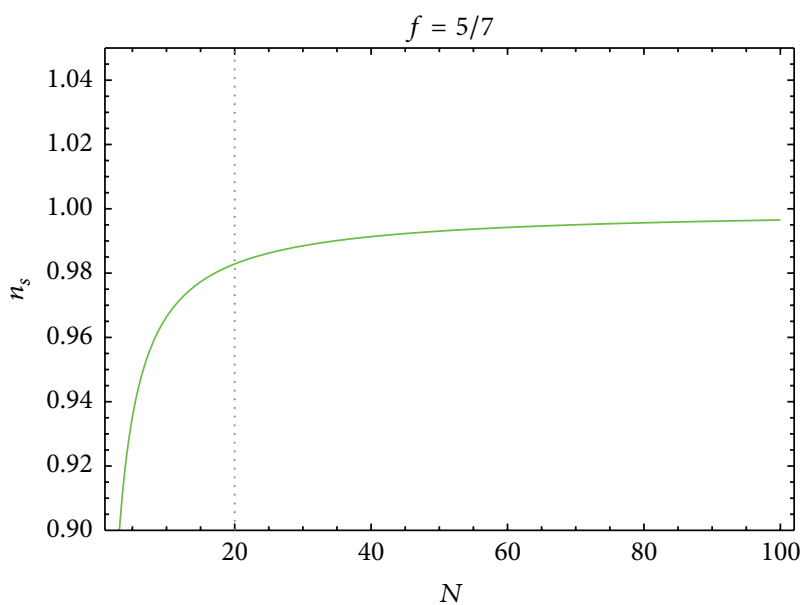

FIGURE 1: In this graph we plot the spectral index $n_{s}$ in terms of the number of e-folds $N$. We can find best range of spectral index ratio where $N>60$.

Harrison-Zeldovich spectrum, that is, $n_{s}=1$, is obtained for an exact value of parameter $f$ (i.e., $f=11 / 14$ ). For $f<11 / 14$ we found the $n_{s}<1$ cases which is compatible with observational data.

In Figure 1, we plot the spectral index in terms of number of e-folds, where $f=5 / 7$. For $N>60$ we can see the spectral index is confined to $0.98<n_{s}<1$ which is compatible with Planck data $[3,4]$.

Tensor-scalar ratio of the model in this case is presented by using (60) and (74)

$$
\begin{aligned}
R & =B_{1}\left(\frac{\phi-\phi_{0}}{\beta}\right)^{(-4 f+1) / 2 f} \operatorname{coth}\left[\frac{k}{2 T}\right] \\
& =B_{1}\left(\frac{N}{A}+\frac{1-f}{f A}\right)^{(-4 f+1) / 4 f} \operatorname{coth}\left[\frac{k}{2 T}\right] \\
& =B_{1}\left(\frac{4 f A}{11-14 f}\left(1-n_{s}\right)\right)^{(4 f-1) / 4 f},
\end{aligned}
$$

where $B_{1}=\left(2^{3 / 2}(4 \pi)^{23 / 8} \Gamma_{0}^{1 / 2} \sigma^{1 / 4}(1-f)^{3 / 4} /\right.$ $\left.3^{15 / 8} M_{4}^{31 / 4} \lambda^{15 / 8}(f A)^{3 / 2}\right)(3 f A / 2 \lambda)^{1 / 2}$. In Figure 2, tensorscalar ratio in terms of number of e-folds is plotted, where $f=5 / 6$. We could see $60<N<80$ lead to $R<0.11$ $[3,5,6]$. The expression for the perturbation $\delta \phi$ given by (43) is valid when $T>H$. The choice of the parameters of the model has to be consistent with this condition $T>H$. In Figure 3 we plot $T / H$ in terms of spectral index that shows the model is compatible with observational data in warm inflation limit $T>H$. We also checked the high dissipative condition $\Gamma_{0}>3 \mathrm{HV}$ in Figure 4 that we can see agreement with observational data.

5.2. $\Gamma=\Gamma_{1} V(\phi)$ Case. Dissipative parameter may be considered as a function of scalar field [50-53]. We will study our model in the context of intermediate inflation, where 


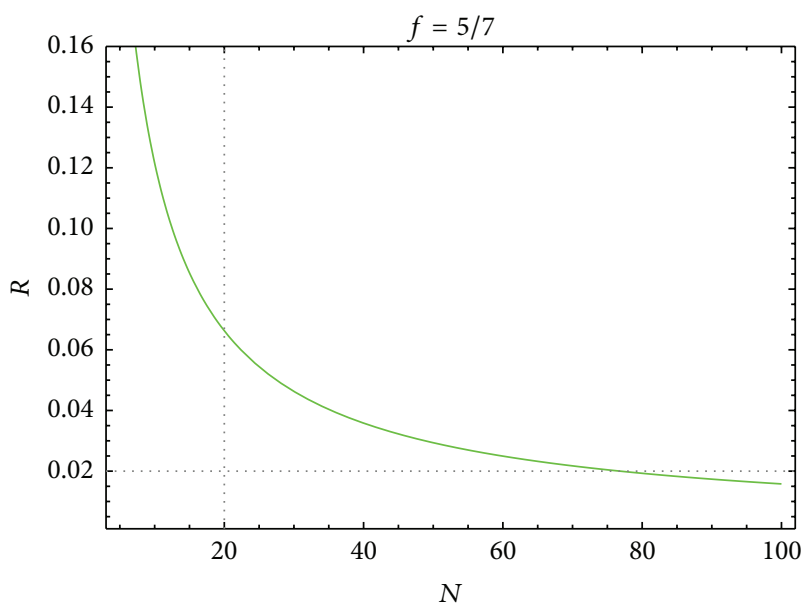

FIgURE 2: In this graph we plot the scalar-tensor ratio $R$ in terms of the number of e-folds $N$. We can find best range of tensor-scalar ratio, where $60<N<80$.

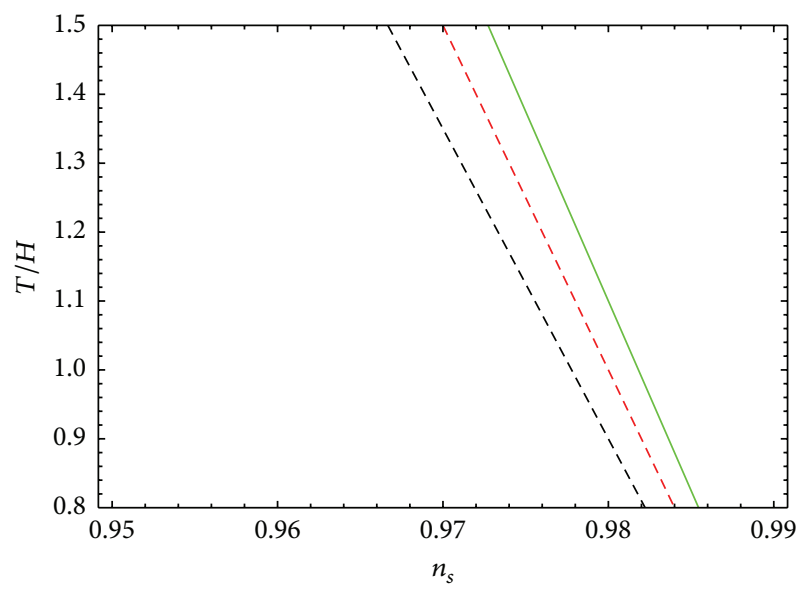

FIGURE 3: In this graph we plot the temperature to Hubble parameter ratio $T / H$ in terms of the spectral index $n_{s}$. We can find best fit of warm inflation condition $(T>H)$ with the Planck data.

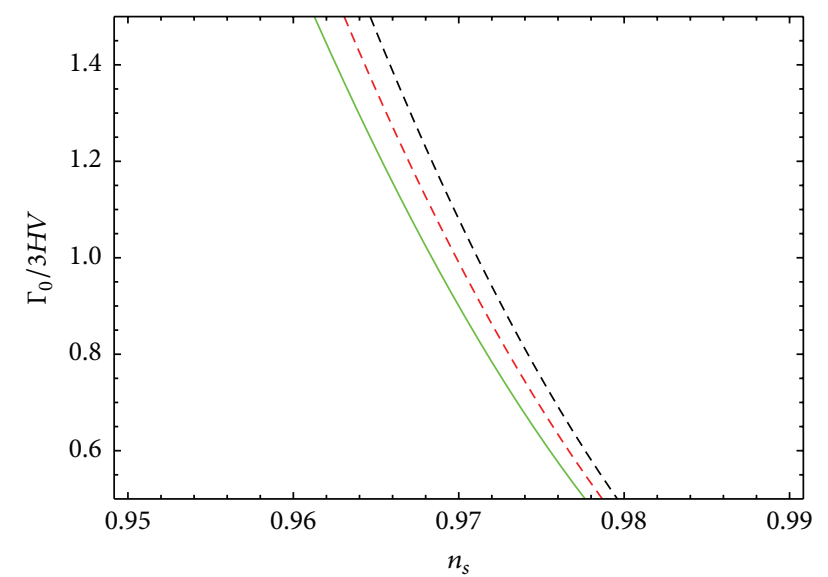

FIGURE 4: In this graph we plot the dissipative to Hubble parameter ratio $\Gamma_{0} / 3 H V$ in terms of the spectral index $n_{s}$. We can find best fit of high dissipative regime $\Gamma_{0}>3 H V$ with the Planck data for three cases of $\Gamma_{0}$.
$\Gamma=\Gamma_{1} V(\phi)$. In this case the scalar field is determined from (74) and (75)

$$
\phi-\phi_{0}=\left(\frac{4(1-f)}{\Gamma_{1}} t\right)^{1 / 2} .
$$

Therefor the Hubble parameter and potential of the model in terms of tachyon potential have the following forms:

$$
\begin{aligned}
& H(\phi)=f A\left(\frac{\Gamma_{1}\left(\phi-\phi_{0}\right)^{2}}{4(1-f)}\right)^{f-1}, \\
& V(\phi)=\left(\frac{3 \lambda M_{4}^{2} f^{2} A^{2}}{4 \pi}\right)^{1 / 2}\left(\frac{\Gamma_{1}\left(\phi-\phi_{0}\right)^{2}}{4(1-f)}\right)^{f-1} .
\end{aligned}
$$

Dissipative parameter $r$ is presented by using above equation

$$
r=\frac{\Gamma_{1} V(\phi)}{3 H V}=\frac{\Gamma_{1}}{f A}\left(\frac{\Gamma_{1}\left(\phi-\phi_{0}\right)^{2}}{4(1-f)}\right)^{1-f} .
$$

Important parameters of the slow-roll inflation in this case are presented as

$$
\begin{gathered}
\epsilon=\frac{1-f}{f A}\left(\frac{\Gamma_{1}\left(\phi-\phi_{0}\right)^{2}}{4(1-f)}\right)^{-f}, \\
\eta=\frac{2-f}{f A}\left(\frac{\Gamma_{1}\left(\phi-\phi_{0}\right)^{2}}{4(1-f)}\right)^{-f} .
\end{gathered}
$$

The number of e-folds is given by

$$
N(\phi)=A\left(\frac{\Gamma_{1}\left(\phi-\phi_{0}\right)^{2}}{4(1-f)}\right)^{f}-A\left(\frac{\Gamma_{1}\left(\phi_{1}-\phi_{0}\right)^{2}}{4(1-f)}\right)^{f},
$$

where $\phi_{1}$ is the tachyon field at the beginning of the inflation period. We find this field where the slow-roll parameter $\epsilon$ is equal to one

$$
\phi_{1}=\phi_{0}+\left[\frac{4(1-f)}{\Gamma_{1}}\left(\frac{1-f}{f A}\right)^{1 / f}\right]^{1 / 2} .
$$

From above equations we present the scalar field in terms of number of e-folds and intermediate parameters $f$ and $A$

$$
\phi=\phi_{0}+\left[\frac{4(1-f)}{\Gamma_{1}}\left(\frac{N}{A}+\frac{1-f}{f A}\right)^{1 / f}\right]^{1 / 2} .
$$

Spectral index $n_{s}$ is presented using (55)

$$
\begin{aligned}
n_{s} & =1+\frac{3}{4} \eta-\frac{23}{4} \epsilon \\
& =1-\frac{17-20 f}{4 f A}\left(\frac{\Gamma_{1}\left(\phi-\phi_{0}\right)^{2}}{4(1-f)}\right)^{-f} \\
& =1-\frac{17-20 f}{4 f A}\left(\frac{N}{A}+\frac{1-f}{f A}\right)^{-1} .
\end{aligned}
$$


We can find the scale invariant spectrum (HarrisonZeldovich spectrum); that is, $n_{s}=1$, where $f=17 / 20$. In Figure 5, we plot the spectral index in terms of number of efolds where $f=5 / 6$. For $N>60$ we can see the spectral index is confined to $0.98<n_{s}<1$ which is compatible with Planck data $[3,4]$. Power spectrum and scalar-tensor ratio of this model may be obtained from (51) and (60), respectively,

$$
\begin{aligned}
P_{R} & =\frac{25}{4} \delta_{H}^{2}=\frac{M_{4}^{5} \lambda^{1 / 2}}{(2 \pi)^{5 / 2} \sigma^{1 / 4}} \frac{V^{15 / 4}}{r^{1 / 2} \epsilon^{3 / 4}} \\
& =A_{2}\left(\frac{\Gamma_{1}\left(\phi-\phi_{0}\right)^{2}}{4(1-f)}\right)^{(20 f-17) / 4 f} \\
& =A_{2}\left(\frac{N}{A}+\frac{1-f}{f A}\right)^{(20 f-17) / 4 f} \\
R & =B_{2}\left(\frac{\Gamma_{1}\left(\phi-\phi_{0}\right)^{2}}{4(1-f)}\right)^{(-10 f+7) / 4 f} \operatorname{coth}\left[\frac{k}{2 T}\right] \\
& =B_{2}\left(\frac{N}{A}+\frac{1-f}{f A}\right)^{(-10 f+7) / 4 f} \\
& =B_{2}\left(\frac{f A\left(1-n_{s}\right)}{20 f-17}\right)^{(10 f-7) / 4 f},
\end{aligned}
$$

where

$$
\begin{aligned}
A_{2} & =\frac{M_{4}^{25 / 4} \lambda^{17 / 4}(f A)^{5} 3^{15 / 8}}{\sigma^{1 / 4} \Gamma_{1}^{1 / 2}(1-f)^{3 / 4} \pi^{35 / 8} 2^{25 / 4}} \\
B_{2} & =\left(\frac{3 f A}{2 \lambda}\right)^{1 / 2} \frac{2^{39 / 8}(2 \pi)^{13 / 8} \sigma^{1 / 4} \Gamma_{1}^{1 / 2}(1-f)^{3 / 4}}{3^{15 / 8} \lambda^{19 / 8} M_{4}^{35 / 8}(f A)^{3}} \\
\mathfrak{J}(\phi) & =-\frac{9}{4} \ln (V) .
\end{aligned}
$$

In Figure 6 we can see high dissipative condition agrees with Planck data. In Figure 7 tensor-scalar ratio in terms of number of e-folds is plotted, where $f=5 / 6$. We could see $60<N$ lead to $R<0.11[3,5,6]$.

\section{Logamediate Inflation}

In this section we will study warm tachyon inflation model in the context of logamediate scenario. The scale factor of this model is given by

$$
a(t)=a_{0} \exp \left(A[\ln t]^{\nu}\right),
$$

where $A$ is a positive constant and $v>1$. We consider this model in two cases: (1) Dissipative parameter $\Gamma$ is constant. (2) Dissipative parameter is proportional to tachyon field potential $V(\phi)$.

6.1. $\Gamma=\Gamma_{0}$ Case. In this case the scalar field is given by using (75) and (95)

$$
\phi-\phi_{0}=\frac{2 \omega}{v+1}(\ln t)^{(\nu+1) / 2},
$$

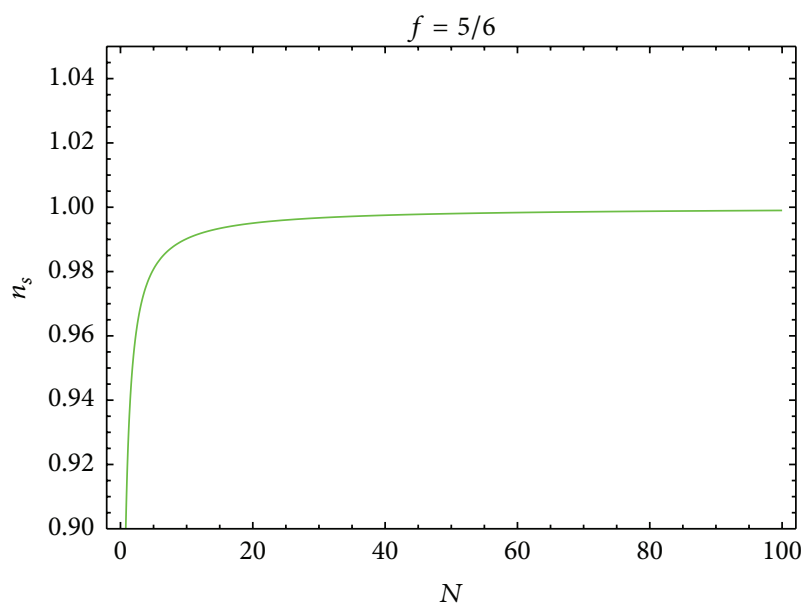

FIGURE 5: In this graph we plot the spectral index $n_{s}$ in terms of the number of e-folds $N$. We can find best range of spectral index ratio, where $N>60$.

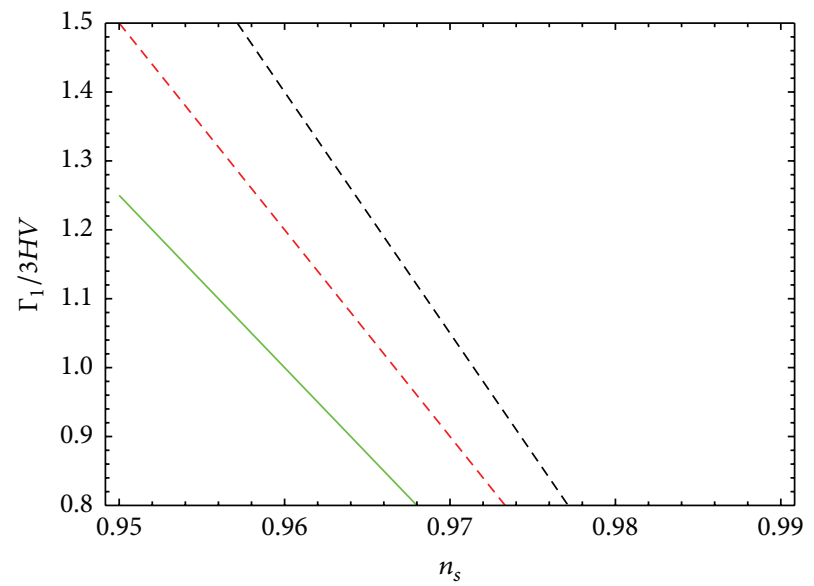

FIGURE 6: In this graph we plot the dissipation to Hubble parameter ratio $\Gamma_{1} / 3 H$ in terms of the spectral index $n_{s}$. We can find best fit of high dissipative regime $\Gamma_{0}>3 H$ with the Planck data for three cases of $\Gamma_{1}$.

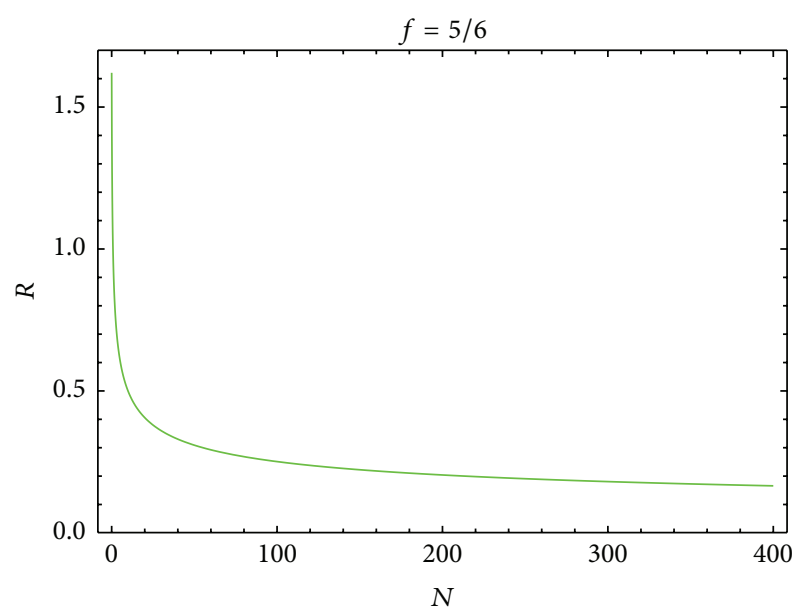

FIGURE 7: In this graph we plot the scalar-tensor ratio $R$ in terms of the number of e-folds $N$. We can find best range of tensor-scalar ratio, where $60<N$. 
where $\omega=\left(3 \lambda M_{4}^{2} \nu^{2} A^{2} / 2 \pi \Gamma_{0}^{2}\right)^{1 / 4}$. Using above equation, the Hubble parameter and tachyon potential have the following forms

$$
\begin{aligned}
& H=\frac{A \nu\left[(\nu+1)\left(\phi-\phi_{0}\right) / 2 \omega\right]^{2(\nu-1) /(\nu+1)}}{\exp \left(\left[(\nu+1)\left(\phi-\phi_{0}\right) / 2 \omega\right]^{2 /(\nu+1)}\right)}, \\
& V=\frac{\Gamma_{0} \omega^{2}\left[(\nu+1)\left(\phi-\phi_{0}\right) / 2 \omega\right]^{2(\nu-1) /(\nu+1)}}{\exp \left(\left[(\nu+1)\left(\phi-\phi_{0}\right) / 2 \omega\right]^{2 /(\nu+1)}\right)} .
\end{aligned}
$$

We derive the slow-roll parameters in logamediate scenario

$$
\begin{aligned}
& \epsilon=\frac{1}{A \nu}\left[\frac{(\nu+1)\left(\phi-\phi_{0}\right)}{2 \omega}\right]^{2(1-\nu) /(\nu+1)}, \\
& \eta=\frac{2}{A \nu}\left[\frac{(\nu+1)\left(\phi-\phi_{0}\right)}{2 \omega}\right]^{2(1-\nu) /(\nu+1)} .
\end{aligned}
$$

The number of e-folds for present model of inflation is presented as:

$$
\begin{aligned}
N & =A\left([\ln t]^{\nu}-\left[\ln t_{1}\right]^{\nu}\right) \\
& =A\left(\left[\frac{(\nu+1)\left(\phi-\phi_{0}\right)}{2 \omega}\right]^{2 v /(\nu+1)}\right. \\
& \left.-\left[\frac{(\nu+1)\left(\phi_{1}-\phi_{0}\right)}{2 \omega}\right]^{2 v /(\nu+1)}\right)
\end{aligned}
$$

$\phi_{1}=\phi_{0}+(2 \omega /(\nu+1))(A \nu)^{(1+\nu) / 2(1-\nu)}$ is the inflation at the beginning of the inflation era. From above equation the scalar field is presented in terms of number of e-folds

$$
\phi=\phi_{0}+\frac{2 \omega}{v+1}\left(\frac{N}{A}+(\nu A)^{v /(1-v)}\right)^{(\nu+1) / 2 v}
$$

Dissipative parameter $r$ is given by

$$
\begin{aligned}
r & =\frac{\Gamma_{0}}{3 H V} \\
& =\frac{1}{3(\nu A \omega)^{2}} \frac{\exp \left(2\left[(\nu+1)\left(\phi-\phi_{0}\right) / 2 \omega\right]^{2 /(\nu+1)}\right)}{\left[(\nu+1)\left(\phi-\phi_{0}\right) / 2 \omega\right]^{4(\nu-1) /(\nu+1)}} .
\end{aligned}
$$

Power spectrum and scalar-tensor ratio of logamediate inflation are derived from (51) and (60):

$$
\begin{aligned}
& P_{R}=A_{3} \exp \left(-\frac{11}{4}\left[\frac{(\nu+1)\left(\phi-\phi_{0}\right)}{2 \omega}\right]^{2 /(\nu+1)}\right) \\
& {\left[\frac{(\nu+1)\left(\phi-\phi_{0}\right)}{2 \omega}\right]^{7(\nu-1) /(\nu+1)}=A_{3}} \\
& \cdot \exp \left(-\frac{11}{4}\left(\frac{N}{A}+(A \nu)^{\nu /(1-\nu)}\right)^{1 / \nu}\right) \\
& \cdot\left[\frac{N}{A}+(A v)^{v /(1-v)}\right]^{7(v-1) / 2 v} \text {, } \\
& R=B_{3} \exp \left(\frac{1}{4}\left[\frac{(\nu+1)\left(\phi-\phi_{0}\right)}{2 \omega}\right]^{2 /(\nu+1)}\right) \\
& \cdot\left[\frac{(\nu+1)\left(\phi-\phi_{0}\right)}{2 \omega}\right]^{4(1-\nu) /(\nu+1)}=B_{3} \\
& \cdot \exp \left(\frac{1}{4}\left(\frac{N}{A}+(A v)^{v /(1-v)}\right)^{1 / v}\right) \\
& \cdot\left[\frac{N}{A}+(A v)^{v /(1-v)}\right]^{4(1-v) / 2 v},
\end{aligned}
$$

where

$$
\begin{aligned}
A_{3} & =\frac{M_{4}^{5} \lambda^{1 / 2} \Gamma_{0}^{7 / 4} \omega^{9 / 2}}{(2 \pi)^{5 / 2} \sigma^{1 / 2}(\nu A)^{-7 / 4}} \\
B_{3} & =\left(\frac{3 \pi \Gamma_{0} \omega}{\lambda^{3} M_{4}^{2}}\right)^{1 / 4} \frac{16(2 \pi)^{5 / 2} \sigma^{1 / 4} \Gamma_{0}^{1 / 4}}{3(3 \omega)^{1 / 2}(A \nu)^{7 / 4}} .
\end{aligned}
$$

By using (55), we could find the spectral index $n_{s}$

$$
\begin{aligned}
n_{s} & =1-\frac{11}{4 \nu A}\left[\frac{(\nu+1)\left(\phi-\phi_{0}\right)}{2 \omega}\right]^{2(1-\nu) /(\nu+1)} \\
& =1-\frac{11}{4 \nu A}\left[\frac{N}{A}+(A \nu)^{\nu /(1-\nu)}\right]^{(1-\nu) / \nu} .
\end{aligned}
$$

In Figure 8, the dependence of spectral index on the number of e-folds is shown (for $v=50$ and $v=5$ cases). It is observed that the small values of the number of e-folds are assured for large values of $v$ parameter. This figure shows the scale invariant spectrum (Harrison-Zeldovich spectrum, i.e., $\left.n_{s}=1\right)$ could be approximately obtained for $(\nu, N)=(50,60)$. From above equation and (102), a relation between scalartensor ratio and spectral index is obtained

$$
\begin{aligned}
R & =B_{3} \exp \left(\frac{1}{4}\left(\frac{4 v A}{11}\left[1-n_{s}\right]\right)^{1 /(1-v)}\right) \\
& \cdot\left[\frac{4 v A}{11}\left(1-n_{s}\right)\right]^{2} .
\end{aligned}
$$

In Figure 9, two trajectories in the $n_{s}-R$ plane are shown. There is a range of values of $R$ and $n_{s}$ which is compatible with the Planck data. 
6.2. $\Gamma=\Gamma_{1} V(\phi)$. Warm tachyon inflation in the context of logamediate scenario with dissipation $\Gamma=\Gamma_{1} V(\phi)$ will be studied. In this case we can find the scalar field using (75) and (95)

$$
\phi-\phi_{0}=\frac{2}{\sqrt{\Gamma_{1}}} t^{1 / 2}
$$

We also derive the Hubble parameter tachyonic potential and dissipative parameter $r$ from above equation

$$
\begin{aligned}
& H(\phi)=\frac{4 A \nu\left(\ln \left(\Gamma_{1}\left(\left(\phi-\phi_{0}\right)^{2} / 4\right)\right)\right)^{\nu-1}}{\left(\phi-\phi_{0}\right)^{2}}, \\
& V(\phi) \\
& =\left(\frac{12 \lambda M_{4}^{2} A^{2} \nu^{2}}{\pi}\right)^{1 / 2} \frac{\left(\ln \left(\Gamma_{1}\left(\left(\phi-\phi_{0}\right)^{2} / 4\right)\right)\right)^{\nu-1}}{\left(\phi-\phi_{0}\right)^{2}}, \\
& r=\frac{\Gamma_{1}}{12 A \nu} \frac{\left(\phi-\phi_{0}\right)^{2}}{\left(\ln \left(\Gamma_{1}\left(\left(\phi-\phi_{0}\right)^{2} / 4\right)\right)\right)^{\nu-1}} .
\end{aligned}
$$
tively,

The slow-roll parameters $\epsilon$ and $\eta$ are presented, respec-

$$
\begin{aligned}
& \epsilon=\frac{\left(\ln \left(\Gamma_{1}\left(\left(\phi-\phi_{0}\right)^{2} / 4\right)\right)\right)^{1-\nu}}{A \nu}, \\
& \eta=\frac{2\left(\ln \left(\Gamma_{1}\left(\left(\phi-\phi_{0}\right)^{2} / 4\right)\right)\right)^{1-\nu}}{A \nu} .
\end{aligned}
$$

Number of e-folds at the end of inflation is given by

$$
\begin{aligned}
N & =A\left[\left(\ln \left(\Gamma_{1} \frac{\left(\phi-\phi_{0}\right)^{2}}{4}\right)\right)^{\nu}\right. \\
& \left.-\left(\ln \left(\Gamma_{1} \frac{\left(\phi_{1}-\phi_{0}\right)^{2}}{4}\right)\right)^{\nu}\right],
\end{aligned}
$$

where $\phi_{1}$ is beginning inflation. At the beginning point of inflation period we have $\epsilon=1$; therefore the inflation in this point has the following form:

$$
\phi_{1}=\phi_{0}+\frac{2}{\sqrt{\Gamma_{1}}} \exp \left(\frac{1}{2}(A \nu)^{v /(1-v)}\right) .
$$

Using above equation we could find the scalar field in terms of number of e-folds

$$
\phi_{1}=\phi_{0}+\frac{2}{\sqrt{\Gamma_{1}}} \exp \left(\frac{1}{2}\left[(A \nu)^{\nu /(1-\nu)}+\frac{N}{A}\right]^{1 / \nu}\right) .
$$

Important perturbation parameters $P_{R}$ (power spectrum) and $R$ (scalar-tensor ratio) could be derived in terms of scalar field and number of e-folds

$$
\begin{aligned}
P_{R} & =A_{4}\left(\phi-\phi_{0}\right)^{-17 / 2}\left[\ln \left(\Gamma_{1} \frac{\left(\phi-\phi_{0}\right)^{2}}{4}\right)\right]^{(20 \nu-9) / 4} \\
& =A_{4}\left(\frac{\sqrt{\Gamma_{1}}}{2}\right)^{17 / 2} \\
& \cdot \exp \left(-\frac{17}{4}\left[\frac{N}{A}+(A \nu)^{\nu /(1-v)}\right]^{1 / \nu}\right) \\
& \cdot\left[\frac{N}{A}+(A v)^{\nu /(1-v)}\right]^{(20 \nu-9) / 4 v}, \\
R & =B_{4}\left(\phi-\phi_{0}\right)^{7 / 2}\left[\ln \left(\Gamma_{1} \frac{\left(\phi-\phi_{0}\right)^{2}}{4}\right)\right]^{(-5 v+5) / 2} \\
& =B_{4}\left(\frac{2}{\sqrt{\Gamma_{1}}}\right)^{7 / 2} \exp \left(\frac{7}{4}\left[\frac{N}{A}+(A \nu)^{\nu /(1-\nu)}\right]^{1 / \nu}\right) \\
& \cdot\left[\frac{N}{A}+(A \nu)^{\nu /(1-v)}\right]^{(-5 \nu+5) / 2 v},
\end{aligned}
$$

where

$$
\begin{aligned}
& A_{4}=\frac{3^{19 / 8} 4^{9 / 8} M_{4}^{35 / 4}(A \nu)^{5}}{\pi^{35 / 8} \sigma^{1 / 4} \lambda^{-19 / 8}} \\
& B_{4}=\frac{4^{1 / 4} \pi^{27 / 8} \sigma^{1 / 4}(A \nu)^{-3}}{3^{19 / 8} M_{4}^{35 / 4} \Gamma_{1}^{1 / 2} \lambda^{19 / 8}}\left(\frac{36 A^{2} \nu^{2}}{\pi \lambda^{2}}\right)^{1 / 4} .
\end{aligned}
$$

The spectral index $n_{s}$ is derived in this case as

$$
\begin{aligned}
n_{s} & =1-\frac{17}{4 A \nu}\left(\ln \left[\frac{\Gamma_{1}\left(\phi-\phi_{0}\right)^{2}}{4}\right]\right) \\
& =1-\frac{17}{8 A \nu}\left[\frac{N}{A}+(\nu A)^{\nu /(1-\nu)}\right]^{1 / \nu} .
\end{aligned}
$$

In Figure 10, the dependence of spectral index on the number of e-folds is shown (for $v=50$ and $v=5$ cases). It is observed that the small values of number of e-folds are assured for large values of $v$ parameter. This figure shows the scale invariant spectrum (Harrison-Zeldovich spectrum, i.e., $n_{s}=1$ ) could be approximately obtained for $(\nu, N)=(50,60)$. From above equation and (112), we find the tensor-scalar ratio in terms of spectral index

$$
\begin{aligned}
R\left(n_{s}\right)= & B_{4}\left(\frac{4}{\Gamma_{1}}\right)^{7 / 4} \exp \left(\frac{7}{4}\left[\frac{4 A \nu}{17}\left(1-n_{s}\right)\right]^{1 /(1-\nu)}\right) \\
& \cdot\left[\frac{4 A \nu}{17}\left(1-n_{s}\right)\right]^{5 / 2} .
\end{aligned}
$$

In Figure 11, two trajectories in the $n_{s}-R$ plane are shown. There is a range of values of $R$ and $n_{s}$ which is compatible with 


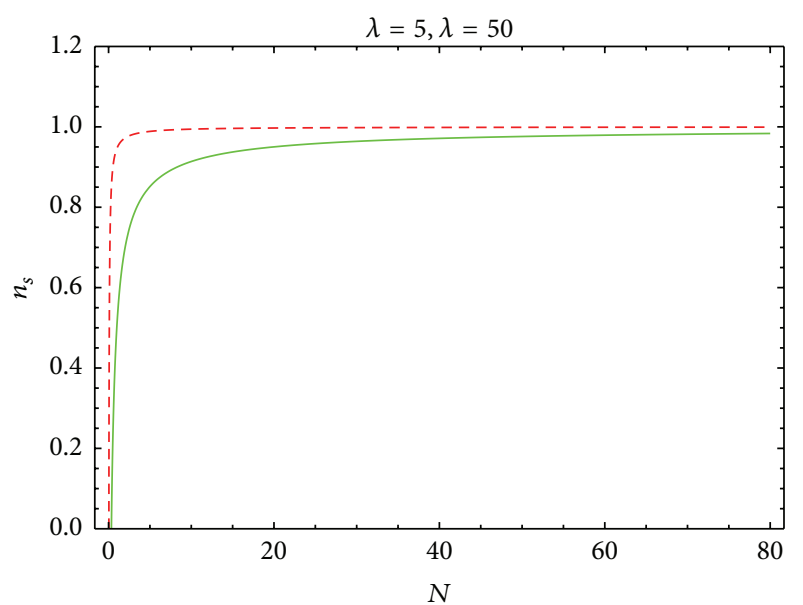

FIGURE 8: Spectral index in terms of number of e-folds, $v=50$ by dashed line and $\nu=5$ by green line.

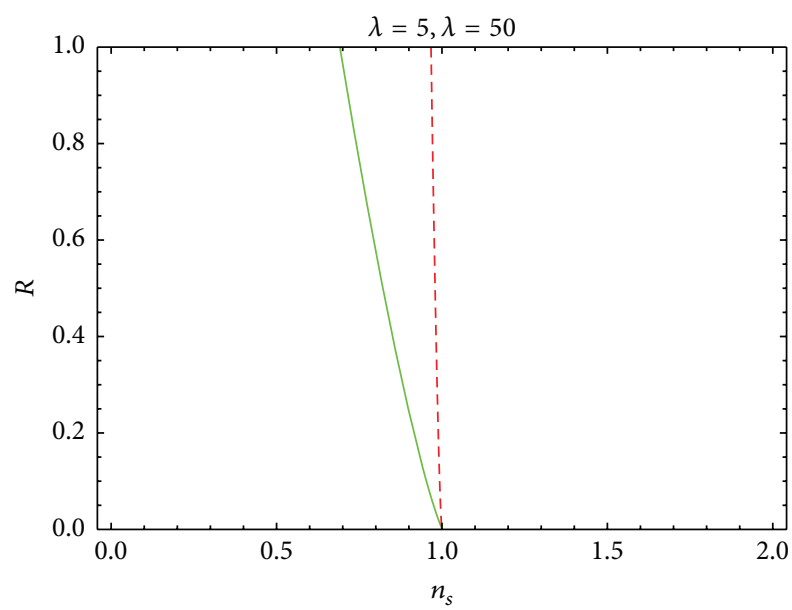

FIGURE 9: Tensor-scalar ratio in terms of spectral index $n_{s}, v=50$ by dashed line and $v=5$ by green line.

the Planck data. In order to produce our plots, we assume some values for the several parameters $\left(f, A, v, \lambda, \Gamma_{0}, \Gamma_{1}\right)$ for the above cases studied, these parameters coincide with $1 \sigma$ confidence level of Planck data. We will use a new method to constrain the parameters of the model in future works. In Figure 12 we plot the tachyonic potential in terms of the spectral index $n_{s}$ in logamediate case. We can find the best fit of high energy limit $V \gg \lambda$ with the Planck data that we have used in this paper.

\section{Conclusion and Discussion}

Tachyon inflation model on the brane with everlasting form of potential $V(\phi)=V_{0} \exp (-\alpha \phi)$ which agrees with tachyon potential properties has been studied. The main problem of the inflation theory is how to attach the universe to the end of the inflation period. One of the solutions of this problem is the study of inflation in the context of warm inflation $[11,12]$. In this scenario radiation is produced during inflation

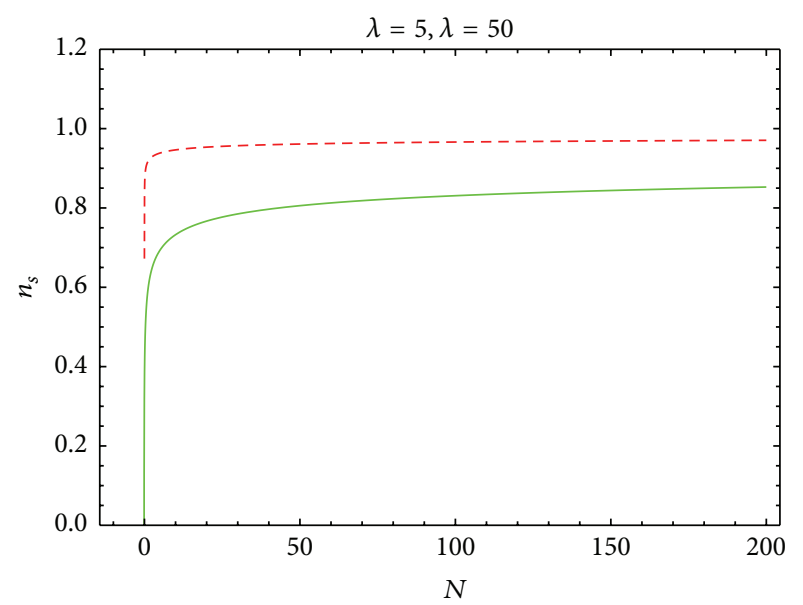

FIGURE 10: Spectral index in terms of number of e-folds, $v=50$ by dashed line and $v=5$ by green line.

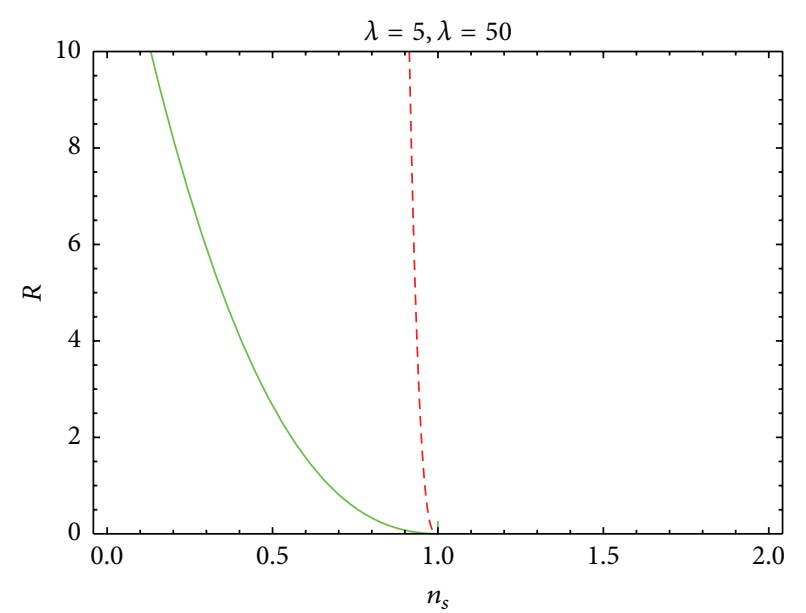

FIGURE 11: Tensor-scalar ratio in terms of spectral index $n_{s}, v=50$ by dashed line and $v=5$ by green line.

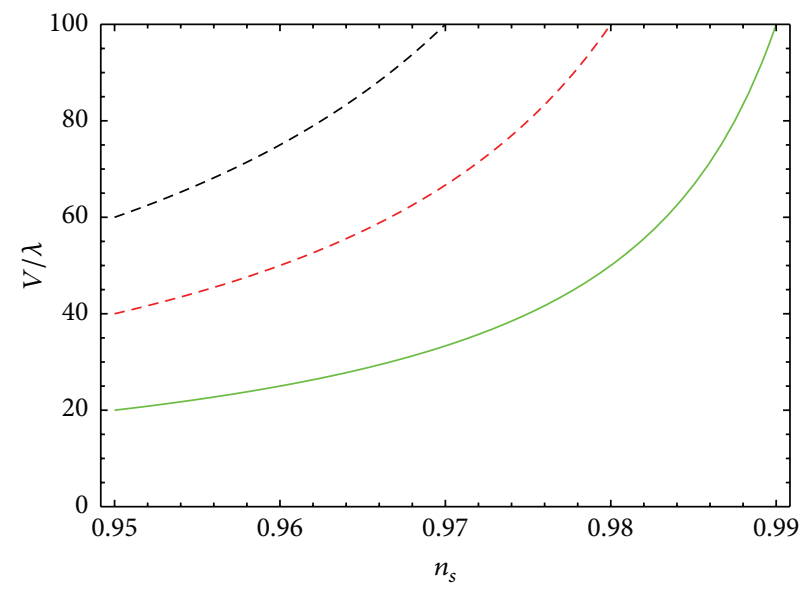

FIGURE 12: In this graph we plot the tachyonic potential in terms of the spectral index $n_{s}$. We can find best fit of high energy limit $V \gg \lambda$ with the Planck data. 
period where its energy density is kept nearly constant. This is phenomenologically fulfilled by introducing the dissipation term $\Gamma$. The study of warm inflation model as a mechanism that gives an end for the tachyon inflation motivated us to consider the warm tachyon inflation model. We note that the $\mathfrak{J}(\phi)$ factor (47) which appears in the perturbation parameters (51), (55), (57), and (60) in high energy limit $(V \gg$ $\lambda)$, for warm tachyon inflation model on the brane, has an important difference with the same factor which was obtained for usual warm tachyon inflation model [50-53]

$$
\begin{aligned}
& \Im(\phi)=-\int\left[\frac{(\Gamma / V)^{\prime}}{3 H+\Gamma / V}+\left(\frac{9}{8}\right.\right. \\
& \cdot \frac{2 H+\Gamma / V}{(3 H+\Gamma / V)^{2}}\left(\Gamma+4 H V-\frac{\Gamma^{\prime}(\ln V)^{\prime}}{12 H(3 H+\Gamma / V)}\right) \\
& \left.\left.\quad \frac{(\ln V)^{\prime}}{V}\right)\right] d \phi .
\end{aligned}
$$

The density square term in the effective Einstein equation on the brane is responsible for this difference. Therefore, the perturbation parameters which may be constrained by Planck observational data are modified due to the effect of density square term in effective Einstein equation. Also the slow-roll parameters (19) and (20) which are derived in the background level are modified because of the density square term in modified Friedmann equation (15). The slow-roll parameters appeared in the perturbation parameters (51), (55), (57), (59), and (60). As have been shown in [50-53] the slow-roll parameters of warm tachyon inflation model have the forms

$$
\begin{aligned}
& \epsilon=\frac{M_{4}^{2}}{16 \pi} \frac{1}{1+r}\left[\frac{V^{\prime}}{V}\right]^{2} \frac{1}{V}, \\
& \eta=\frac{M_{4}^{2}}{8 \pi(1+r) V}\left[\frac{V^{\prime \prime}}{V}-\frac{1}{2}\left(\frac{V^{\prime}}{V}\right)^{2}\right] .
\end{aligned}
$$

These parameters are obviously different from the slow-roll parameters (19) and (20). Perturbation parameters of warm tachyon inflation model have following from [50-53]:

$$
\begin{aligned}
& \delta_{H}=\frac{\sqrt{3}}{75 \pi^{2}} \frac{\exp (-2 \mathfrak{\Im}(\phi))}{r^{1 / 2} \widetilde{\epsilon}}, \\
& n_{s}=1-\left[\frac{3 \widetilde{\eta}}{2}+\widetilde{\epsilon}\left(\frac{2 V^{\prime}}{V}\left[2 \widetilde{\Im}^{\prime}(\phi)-\frac{r^{\prime}}{4 r}\right]-\frac{5}{2}\right)\right], \\
& \alpha_{s}=\frac{2 V}{V^{\prime}} \widetilde{\epsilon} n_{s^{\prime}}^{\prime} \\
& n_{g}=-2 \epsilon \\
& R\left(k_{0}\right) \\
& =\left.\frac{240 \sqrt{3}}{25 m_{p}^{2}}\left[\frac{r^{1 / 2} \widetilde{\epsilon} H^{3}}{T_{r}} \exp (2 \widetilde{\Im}(\phi)) \operatorname{coth}\left[\frac{k}{2 T}\right]\right]\right|_{k=k_{0}} .
\end{aligned}
$$

The above parameters are also different from the perturbation parameters of our model on the brane (51), (55), (57), (59), and (60) because of the density square term in the effective Einstein equation on the brane. So, from above discussion, we know the density square term in the effective Einstein equation on the brane gives the significant contributions to the observable parameters, $P_{R}, R, n_{s}$, and $\alpha_{s}$. Also, the different observable perturbation parameters for the models of nontachyon warm inflation and nontachyon warm inflation model on the brane are presented in [49] and [44], respectively.

In tachyon Randall-Sundrum brane world scenario Einstein's equation and therefore the Friedmann equation are modified. Warm tachyon inflation parameters on the brane have important differences with the same parameters which were presented for usual warm inflation model [44] because of this modification. The density square term in the effective Einstein equation on the brane is responsible for this difference. Therefore, the perturbation parameters which may be constrained by Planck observational data are modified due to the effect of density square term in effective Einstein equation and modification of tachyonic scalar field equation of motion (EMO) instead of normal scalar fields EMO. In this paper we have considered warm tachyon inflationary universe model on the brane. In the slow-roll approximation the general relation between energy density of radiation and energy density of tachyon field is presented. In the longitudinal gauge and the slow-roll limit the explicit expressions for the tensorscalar ratio $R$ scalar spectrum $P_{R}$ index, $n_{s}$ and its running $\alpha_{s}$, have been presented. We have developed our specific model by exponential potential with a constant dissipation coefficient. In this case we have found perturbation parameters and constrained these parameters Planck observational data. Intermediate and logamediate inflation are considered for two cases of dissipative parameters: (1) $\Gamma$ is constant parameter. (2) $\Gamma$ is a function of tachyon field. In these two cases we have found that the models are compatible with observational data. Harrison-Zeldovich spectrum, that is, $n_{s}=1$, is obtained exactly by one parameter in intermediate scenario $\left(f=11 / 14\right.$ for $\Gamma=\Gamma_{0}$ case and $f=17 / 20$ for $\Gamma=\Gamma(\phi))$. In logamediate scenario we have presented approximately scale invariant spectrum; that is, $n \simeq 1$, where $(N, v)=(60,50)$.

\section{Appendix}

In this paper we have studied the model in natural unit $(h / 2 \pi=c=1)$; therefore we have $([$ mass $]=M$, [ time $]=T$, and [length] $=L$, where $[A]$ means dimension of " $A$ ")

$$
\begin{gathered}
{[c]=L T^{-1}=1,} \\
{[h]=M L^{2} T^{-1}} \\
\Downarrow
\end{gathered}
$$

$$
T=L=M^{-1}
$$


Using (7) we have

$$
\begin{aligned}
{\left[H^{2}\right] } & =\left[\frac{8 \pi}{M_{4}^{2}} \rho_{T}\left(1+\frac{\rho_{T}}{2 \lambda}\right)\right] \Longrightarrow \\
\frac{\left[a^{2}\right]}{a^{2} T^{2}} & =\frac{\left[\rho_{T}\right]}{\left[M_{4}^{2}\right]} \Longrightarrow \\
{\left[\rho_{T}\right] } & =\left[T_{\mu \nu}\right]=[V]=[P]=M^{4},
\end{aligned}
$$

where $V$ and $P$ are potential and pressure with dimension $M^{4}$. From (11) we have

$$
\begin{aligned}
& {[\dot{\phi}]=1 \Longrightarrow} \\
& {[\phi]=M^{-1} .}
\end{aligned}
$$

It appears that tachyon scalar field has dimension $M^{-1}$ which agrees with the tachyonic potential (61). In (13) right-hand side and left-hand side have dimension $M^{4}$

$$
\begin{aligned}
{[\dot{\rho}]+[3 H \rho]+[3 H P] } & =\left[\Gamma \dot{\phi}^{2}\right] \Longrightarrow \\
\frac{[\rho]}{T}+\frac{[\rho]}{T}+\frac{[P]}{T} & =[\Gamma] \Longrightarrow \\
{[\Gamma] } & =M^{5} .
\end{aligned}
$$

In (16) we have used dimensionless parameter $r=$ $(\Gamma / V)(1 / 3 H)$

$$
[r]=\frac{[\Gamma]}{[H][V]}=\frac{M^{5}}{M M^{4}}=1 .
$$

$V / \Gamma$ has dimension time $\left(H^{-1}\right)$; therefore in our paper we have used $\Gamma / V$ instead of $\Gamma$. We note that from above discussion that $\chi$ in (41) has dimension $M^{-2}$ which leads to $[C]=M^{-2}$ in (45) and (49) has correct dimension

$$
\begin{aligned}
& {[\delta \phi]=[C] \frac{\left[V^{\prime}\right]}{[V]},} \\
& M^{-1}=M^{-2} \frac{1}{M^{-1}} .
\end{aligned}
$$

In (47), we have $2 H+\Gamma / V$ where the analysis of dimension is given by

$$
[2 H]+\frac{[\Gamma]}{V}=M+\frac{M^{5}}{M^{4}} .
$$

Equation (49) has correct dimension; for cold inflation we have $\left[\delta_{H}\right]=([H] /[\dot{\phi}])[\delta \phi]=1$; in warm inflation also we have from (49)

$$
\delta_{H}=\left[M_{4}^{2}\right] \frac{[V][\delta \phi]}{\left[V^{\prime}\right]}=M^{2} M^{-1} M^{-1}=1 .
$$

We note that $(50)$ is in momentum space $[66,86]$. Hence, inserting (50) into (49) means that (51) and the following equations are in momentum space.

\section{Competing Interests}

The authors declare that they have no competing interests.

\section{References}

[1] A. H. Guth, "Inflationary universe: a possible solution to the horizon and flatness problems," Physical Review D, vol. 23, no. 2, pp. 347-356, 1981.

[2] A. Albrecht and P. J. Steinhardt, "Cosmology for grand unified theories with radiatively induced symmetry breaking," Physical Review Letters, vol. 48, no. 17, pp. 1220-1223, 1982.

[3] P. A. R. Ade, N. Aghanim, M. Arnaud et al., "Planck 2015 results. XX. Constraints on inflation," https://arxiv.org/abs/1502.02114.

[4] G. Hinshaw, D. Larson, E. Komatsu et al., "Nine-year Wilkinson microwave anisotropy probe (WMAP) observations: cosmological parameter results," The Astrophysical Journal Supplement Series, vol. 208, no. 2, p. 19, 2013.

[5] P. A. R. Ade, N. Aghanim, C. Armitage-Caplan et al., "Planck 2013 results. XVI. Cosmological parameters," Astronomy \& Astrophysics, vol. 571, article A16, 2014.

[6] P. A. R. Ade, N. Aghanim, C. Armitage-Caplan et al., "Planck 2013 results. XXII. Constraints on inflation," Astronomy \& Astrophysics, vol. 571, article A22, 2014.

[7] J. H. Traschen and R. H. Brandenberger, "Particle production during out-of-equilibrium phase transitions," Physical Review $D$, vol. 42, no. 8, pp. 2491-2504, 1990.

[8] L. Kofman, A. Linde, and A. A. Starobinsky, "Reheating after inflation," Physical Review Letters, vol. 73, no. 24, pp. 3195-3198, 1994.

[9] Y. Shtanov, J. Traschen, and R. Brandenberger, "Universe reheating after inflation," Physical Review D, vol. 51, no. 10, pp. 5438-5455, 1995.

[10] L. Kofman, A. Linde, and A. A. Starobinsky, "Towards the theory of reheating after inflation," Physical Review D, vol. 56, no. 6, pp. 3258-3295, 1997.

[11] A. Berera, "Warm inflation," Physical Review Letters, vol. 75, no. 18, pp. 3218-3221, 1995.

[12] A. Berera, "Interpolating the stage of exponential expansion in the early universe: possible alternative with no reheating," Physical Review D-Particles, Fields, Gravitation and Cosmology, vol. 55, no. 6, pp. 3346-3357, 1997.

[13] I. G. Moss, "Primordial inflation with spontaneous symmetry breaking," Physics Letters B, vol. 154, no. 2-3, pp. 120-124, 1985.

[14] A. Berera, "Warm inflation in the adiabatic regime-a model, an existence proof for inflationary dynamics in quantum field theory," Nuclear Physics B, vol. 585, no. 3, pp. 666-714, 2000.

[15] Y.-F. Cai, J. B. Dent, and D. A. Easson, "Warm dirac-born-infeld inflation," Physical Review D, vol. 83, no. 10, Article ID 101301, 2011.

[16] R. Cerezo and J. G. Rosa, "Warm inflection," Journal of High Energy Physics, vol. 2013, article 24, 2013.

[17] S. Bartrum, A. Berera, and J. G. Rosa, "Gravitino cosmology in supersymmetric warm inflation," Physical Review D, vol. 86, Article ID 123525, 2012.

[18] M. Bastero-Gil, A. Berera, R. O. Ramos, and J. G. Rosa, "Warm baryogenesis," Physics Letters, Section B: Nuclear, Elementary Particle and High-Energy Physics, vol. 712, no. 4-5, pp. 425-429, 2012. 
[19] M. Bastero-Gil, A. Berera, and J. G. Rosa, "Warming up braneantibrane inflation," Physical Review D, vol. 84, no. 10, Article ID 103503, 2011.

[20] A. Berera, M. Gleiser, and R. O. Ramos, "Strong dissipative behavior in quantum field theory," Physical Review D, vol. 58, Article ID 123508, 1998.

[21] A. Berera, M. Gleiser, and R. O. Ramos, "A first principles warm inflation model that solves the cosmological horizon and flatness problems," Physical Review Letters, vol. 83, no. 2, pp. 264-267, 1999.

[22] M. Bastero-Gil, A. Berera, and R. O. Ramos, "Dissipation coefficients from scalar and fermion quantum field interactions," Journal of Cosmology and Astroparticle Physics, vol. 2011, no. 9, article 033, 2011.

[23] M. Bastero-Gil, A. Berera, R. O. Ramos, and J. G. Rosa, "General dissipation coefficient in low-temperature warm inflation," Journal of Cosmology and Astroparticle Physics, vol. 2013, no. 1, article 016, 2013.

[24] A. Sen, "Rolling Tachyon," Journal of High Energy Physics, vol. 204, p. 48, 2002.

[25] A. Sen, "Field theory of tachyon matter," Modern Physics Letters A, vol. 17, no. 27, pp. 1797-1804, 2002.

[26] M. Sami, P. Chingangbam, and T. Qureshi, "Aspects of tachyonic inflation with an exponential potential," Physical Review D, vol. 66, no. 4, Article ID 043530, 2002.

[27] G. W. Gibbons, "Cosmological evolution of the rolling tachyon," Physics Letters B, vol. 537, no. 1-2, pp. 1-4, 2002.

[28] C. Armendáriz-Picón, T. Damour, and V. Mukhanov, " $k$ Inflation,” Physics Letters B, vol. 458, no. 2-3, pp. 209-218, 1999.

[29] K. Akama, Gauge Theory and Gravitation, vol. 176 of Lecture Notes in Physics, Springer, Berlin, Germany, 1982.

[30] V. A. Rubakov and M. E. Shaposhnikov, "An exotic class of Kaluza-Klein models," Physics Letters B, vol. 159, no. 1, pp. 2225, 1985.

[31] N. Arkani-Hamed, S. Dimopoulos, and G. Dvali, "The hierarchy problem and new dimensions at a millimeter," Physics Letters, Section B: Nuclear, Elementary Particle and High-Energy Physics, vol. 429, no. 3-4, pp. 263-272, 1998.

[32] M. Gogberashvili, "Our world as an expanding shell," Europhysics Letters, vol. 49, no. 3, pp. 396-399, 2000.

[33] L. Randall and R. Sundrum, "Large mass hierarchy from a small extra dimension," Physical Review Letters, vol. 83, no. 17, pp. 3370-3373, 1999.

[34] L. Randall and R. Sundrum, "An alternative to compactification," Physical Review Letters, vol. 83, no. 23, pp. 4690-4693, 1999.

[35] J. Polchinski, "Dirichlet branes and Ramond-Ramond charges," Physical Review Letters, vol. 75, no. 26, pp. 4724-4727, 1995.

[36] P. Horava and E. Witten, "Heterotic and type I string dynamics from eleven dimensions," Nuclear Physics. B, vol. 460, no. 3, pp. 506-524, 1996.

[37] A. Lukas, B. A. Ovrut, and D. Waldram, "Cosmological solutions of Hořava-Witten theory," Physical Review D, vol. 60, no. 8, Article ID 086001, 1999.

[38] T. Shiromizu, K.-I. Maeda, and M. Sasaki, "The Einstein equations on the 3-brane world," Physical Review D, vol. 62, no. 2, Article ID 024012, 2000.

[39] D. Langlois, R. Maartens, M. Sasaki, and D. Wands, "Large-scale cosmological perturbations on the brane," Physical Review D, vol. 63, no. 8, Article ID 084009, 2001.
[40] P. R. Ashcroft, C. van de Bruck, and A.-C. Davis, "Suppression of entropy perturbations in multifield inflation on the brane," Physical Review D, vol. 66, no. 12, Article ID 121302, 5 pages, 2002.

[41] R. Maartens, "Cosmological dynamics on the brane," Physical Review D, vol. 62, no. 8, Article ID 084023, 14 pages, 2000.

[42] C. Gordon and R. Maartens, "Density perturbations in the brane-world," Physical Review D, vol. 63, no. 4, Article ID 044022, 2001.

[43] D. Folini and R. Walder, "Theoretical predictions for the cold part of the colliding wind interaction zone," http://arxiv.org/abs/astro-ph/0012132.

[44] M. A. Cid, S. del Campo, and R. Herrera, "Warm inflation on the brane," Journal of Cosmology and Astroparticle Physics, vol. 2007, no. 10, p. 5, 2007.

[45] J. M. Cline, C. Grojean, and G. Servant, "Cosmological expansion in the presence of an extra dimension," Physical Review Letters, vol. 83, no. 21, pp. 4245-4248, 1999.

[46] P. Brax and C. van de Bruck, "Cosmology and brane worlds: a review," Classical and Quantum Gravity, vol. 20, no. 9, pp. R201R232, 2003.

[47] T. Clifton, P. G. Ferreira, A. Padilla, and C. Skordis, "Modified gravity and cosmology," Physics Reports, vol. 513, no. 1-3, pp. 1189, 2012.

[48] S. del Campo and R. Herrera, "Warm inflation in the DGP brane-world model," Physics Letters B, vol. 653, no. 2-4, pp. 122128, 2007.

[49] H. P. de Oliveira, "Density perturbations in warm inflation and COBE normalization," Physics Letters B, vol. 526, no. 1-2, pp. 1-8, 2002.

[50] R. Herrera, S. del Campo, and C. Campuzano, "Tachyon warm inflationary universe models," Journal of Cosmology and Astroparticle Physics, vol. 2006, no. 10, p. 9, 2006.

[51] M. R. Setare and V. Kamali, "Cosmological perturbations in warm-tachyon inflationary universe model with viscous pressure on the brane," Journal of High Energy Physics, vol. 2013, no. 3, article 066, 2013.

[52] M. R. Setare and V. Kamali, “Tachyon warm-logamediate inflationary universe model in a high dissipative regime," Physical Review D, vol. 87, no. 8, Article ID 083524, 2013.

[53] A. Deshamukhya and S. Panda, "Warm tachyonic inflation in a warped background," International Journal of Modern Physics D, vol. 18, no. 14, pp. 2093-2106, 2009.

[54] T. Clifton and J. D. Barrow, "Decay of the cosmic vacuum energy," https://arxiv.org/abs/1412.5465.

[55] A. Cid, G. Leon, and Y. Leyva, "Intermediate accelerated solutions as generic late-time attractors in a modified JordanBrans-Dicke theor," Journal of Cosmology and Astroparticle Physics, vol. 2016, no. 2, article 027, 2016.

[56] A. K. Sanyal, "If Gauss-Bonnet interaction plays the role of dark energy," Physics Letters B, vol. 645, no. 1, pp. 1-5, 2007.

[57] T. Koivisto and D. F. Mota, "Cosmology and astrophysical constraints of Gauss-Bonnet dark energy," Physics Letters B, vol. 644, no. 2-3, pp. 104-108, 2007.

[58] T. Koivisto and D. F. Mota, "Gauss-Bonnet quintessence: background evolution, large scale structure, and cosmological constraints," Physical Review D, vol. 75, Article ID 023518, 2007.

[59] S. Mignemi and N. R. Stewart, "Charged black holes in effective string theory," Physical Review D, vol. 47, no. 12, pp. 5259-5269, 1993. 
[60] S. Nojiri, S. D. Odintsov, and M. Sasaki, "Gauss-Bonnet dark energy," Physical Review D, vol. 71, no. 12, Article ID 123509, 2005.

[61] G. Cognola, E. Elizalde, S. Nojiri, S. D. Odintsov, and S. Zerbini, "Dark energy in modified Gauss-Bonnet gravity: latetime acceleration and the hierarchy problem," Physical Review $D$, vol. 73, no. 8, Article ID 084007, 2006.

[62] I. Antoniadis, J. Rizos, and K. Tamvakis, "Singularity-free cosmological solutions of the superstring effective action," Nuclear Physics, Section B, vol. 415, no. 2, pp. 497-514, 1994.

[63] J. D. Barrow and A. R. Liddle, "Influence of induced magnetic fields on the static properties of Josephson-junction arrays," Physical Review D, vol. 47, pp. 5219-5229, 1993.

[64] A. Vallinotto, E. J. Copeland, E. W. Kolb, A. R. Liddle, and D. A. Steer, "Inflationary potentials yielding constant scalar perturbation spectral indices," Physical Review D, vol. 69, no. 10, Article ID 103519, 8 pages, 2004.

[65] A. A. Starobinsky, "Inflaton field potential producing an exactly flat spectrum of adiabatic perturbations," JETP Letters, vol. 82, no. 4, pp. 169-173, 2005.

[66] M. R. Setare and V. Kamali, "Tachyon warm-intermediate inflationary universe model in high dissipative regime," Journal of Cosmology and Astroparticle Physics, vol. 2012, no. 8, article 034, 16 pages, 2012.

[67] J. D. Barrow, "Varieties of expanding universe," Classical and Quantum Gravity, vol. 13, no. 11, pp. 2965-2975, 1996.

[68] J. D. Barrow, "Slow-roll inflation in scalar-tensor theories," Physical Review D, vol. 51, p. 2729, 1995.

[69] P. J. E. Peebles and B. Ratra, "The cosmological constant and dark energy," Reviews of Modern Physics, vol. 75, no. 2, pp. 559606, 2003.

[70] P. G. Ferreira and M. Joyce, "Cosmology with a primordial scaling field," Physical Review D, vol. 58, no. 2, Article ID 023503, 1998.

[71] J. D. Barrow and N. J. Nunes, "Dynamics of 'logamediate' inflation," Physical Review D, vol. 76, no. 4, Article ID 043501, 2007.

[72] J. Yokoyama and K. Maeda, "On the dynamics of the power law inflation due to an exponential potential," Physics Letters B, vol. 207, no. 1, pp. 31-35, 1988.

[73] R. Herrera, "Warm inflationary model in loop quantum cosmology," Physical Review D, vol. 81, Article ID 123511, 2010.

[74] K. Xiao and J. Y. Zhu, "A phenomenology analysis of the tachyon warm inflation in loop quantum cosmology," Physics Letters B, vol. 699, no. 4, pp. 217-223, 2011.

[75] R. Herrera and E. San Martin, "Warm-intermediate inflationary universe model in braneworld cosmologies," The European Physical Journal C, vol. 71, article 1701, 2011.

[76] R. Herrera and M. Olivares, "Warm-logamediate inflationary universe model," International Journal of Modern Physics D, vol. 21, no. 5, Article ID 1250047, 13 pages, 2012.

[77] M. R. Setare and V. Kamali, "Cosmological perturbations in warm-tachyon inflationary universe model with viscous pressure on the brane," Journal of High Energy Physics, vol. 2013, no. 3, article 066, 2013.

[78] A. Cid, "On the consistency of tachyon warm inflation with viscous pressure," Physics Letters B, vol. 743, pp. 127-133, 2015.

[79] A. Sen, "Tachyon condensation on the brane antibrane system," Journal of High Energy Physics, vol. 1998, no. 08, 1998.
[80] J. M. Bardeen, “Gauge-invariant cosmological perturbations," Physical Review. D. Particles and Fields. Third Series, vol. 22, no. 8, pp. 1882-1905, 1980.

[81] V. F. Mukhanov, H. A. Feldman, and R. H. Brandenberger, "Theory of cosmological perturbations," Physics Reports A: Review Section of Physics Letters, vol. 215, no. 5-6, pp. 203-333, 1992.

[82] A. A. Starobinsky and J. Yokoyama, "Density fluctuations in Brans-Dicke inflation," in Proceedings of the 4th Workshop on General Relativity and Gravitation (JGRG '94), p. 381, 1994, https://inspirehep.net/search?p=find+eprint+GR-QC/9502002.

[83] A. A. Starobinsky, S. Tsujikawa, and J. Yokoyama, "Cosmological perturbations from multi-field inflation in generalized Einstein theories," Nuclear Physics. B, vol. 610, no. 1-2, pp. 383410, 2001.

[84] H. P. de Oliveira and S. E. Jorás, "Perturbations in warm inflation," Physical Review D, vol. 64, Article ID 063513, 2001.

[85] L. M. H. Hall, I. G. Moss, and A. Berera, "Scalar perturbation spectra from warm inflation," Physical Review D, vol. 69, no. 8, Article ID 083525, 2004.

[86] A. N. Taylor and A. Berera, "Perturbation spectra in the warm inflationary scenario," Physical Review D, vol. 62, no. 8, Article ID 083517, 2000.

[87] V. N. Lukash, "Production of phonons in an isotropic universe," Soviet Physics-JETP, vol. 52, pp. 807-814, 1980.

[88] H. Kodama and M. Sasaki, "Cosmological perturbation theory," Progress of Theoretical Physics Supplement, vol. 78, pp. 1-166, 1984.

[89] J. E. Lidsey, A. R. Liddle, E. W. Kolb, E. J. Copeland, T. Barreiro, and M. Abney, "Reconstructing the inflaton potential-an overview," Reviews of Modern Physics, vol. 69, no. 2, pp. 373-410, 1997.

[90] B. A. Bassett, S. Tsujikawa, and D. Wands, "Inflation dynamics and reheating," Reviews of Modern Physics, vol. 78, no. 2, pp. 537-589, 2006.

[91] D. Langlois, R. Maartens, and D. Wands, "Gravitational waves from inflation on the brane," Physics Letters B, vol. 489, no. 3-4, pp. 259-267, 2000.

[92] R. Herrera, N. Videla, and M. Olivares, "Warm intermediate inflation in the Randall-Sundrum II model in the light of Planck 2015 and BICEP2 results: a general dissipative coefficient," The European Physical Journal C, vol. 75, article 205, 2015.

[93] K. Bhattacharya, S. Mohanty, and A. Nautiyal, "Enhanced polarization of the cosmic microwave background radiation from thermal gravitational waves," Physical Review Letters, vol. 97, no. 25, Article ID 251301, 2006.

[94] M. Fairbairn and M. H. Tytgat, "Inflation from a tachyon fluid?" Physics Letters B, vol. 546, no. 1-2, pp. 1-7, 2002. 

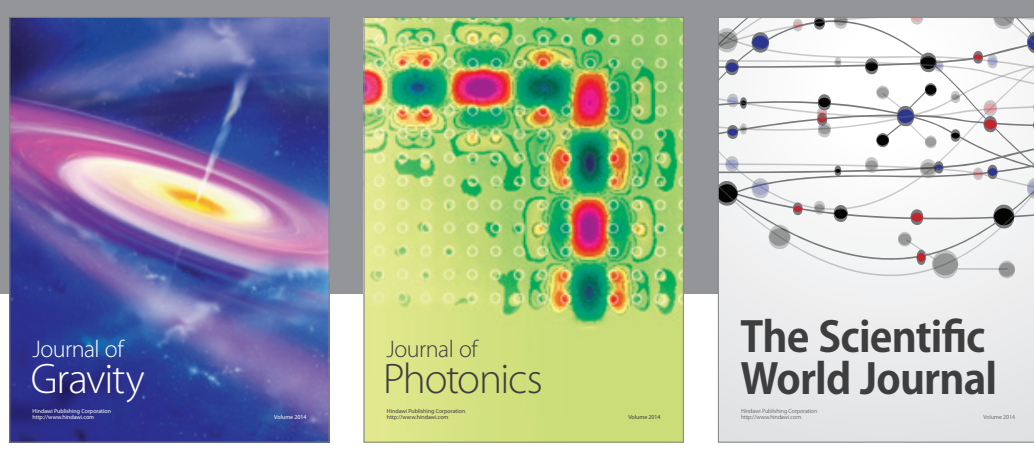

The Scientific World Journal
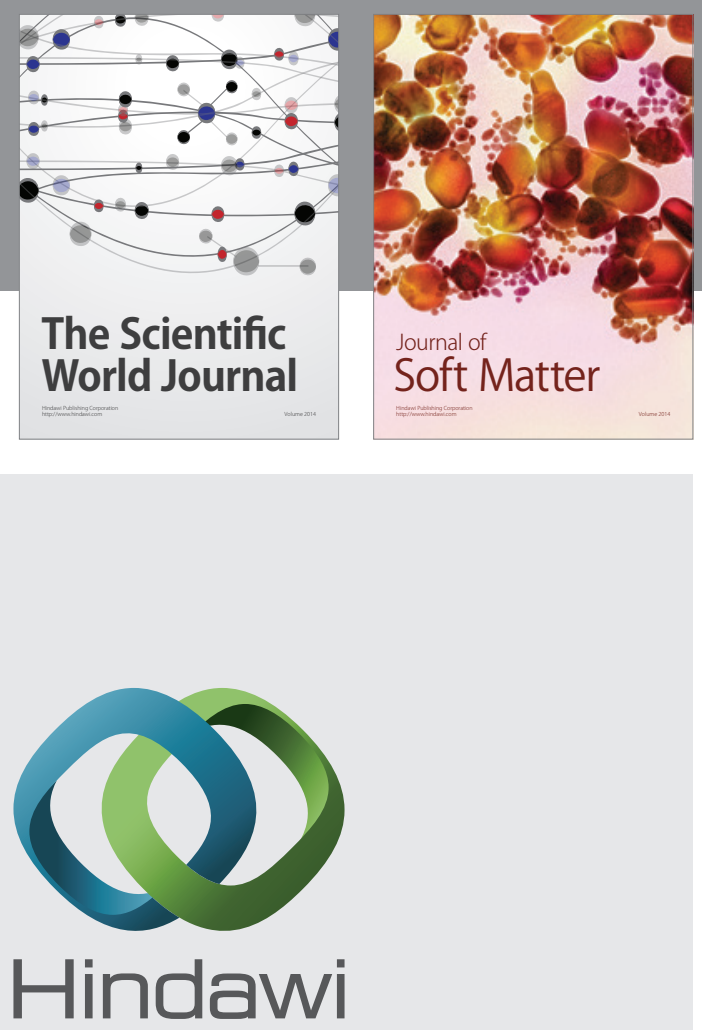

Submit your manuscripts at

http://www.hindawi.com

nternational Journal of

Statistical Mechanics
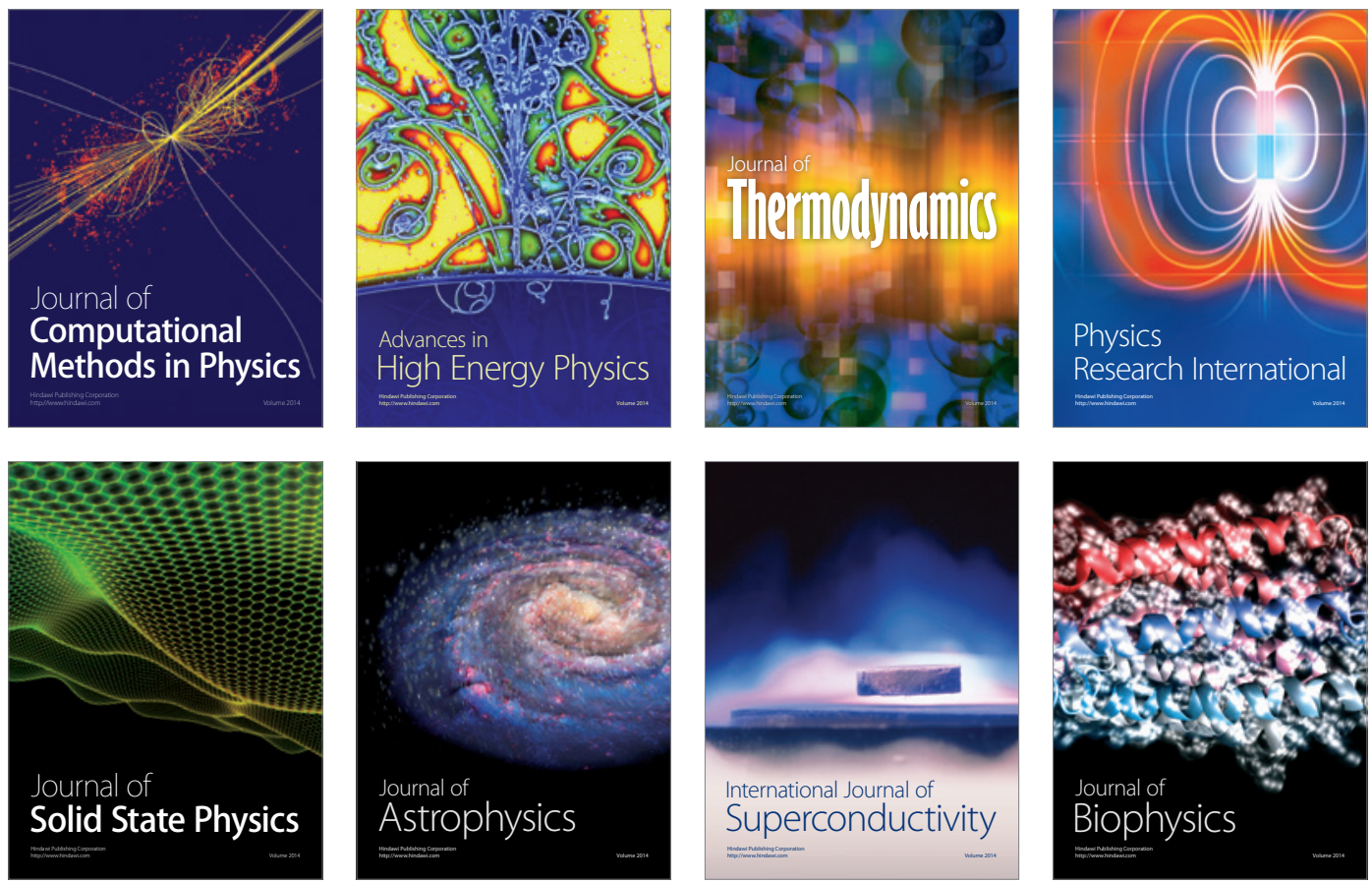
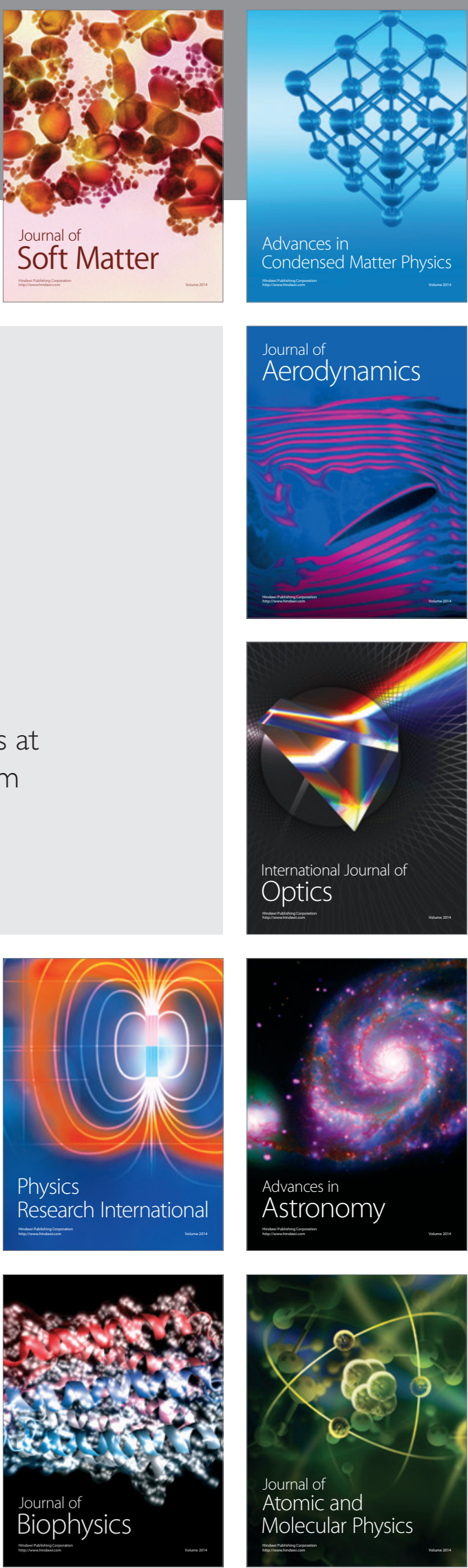